DRAFT VERSION DECEMBER 23, 2020

Typeset using $\mathrm{LAT}_{\mathrm{E}} \mathrm{X}$ default style in AASTeX63

\title{
Effect of acceleration and escape of energetic particles on spectral steepening at shocks
}

\author{
FEDERICO FRASCHETTI ${ }^{1,2}$ \\ ${ }^{1}$ Department of Planetary Sciences-Lunar and Planetary Laboratory, University of Arizona, Tucson, AZ, 85721, USA \\ ${ }^{2}$ Center for Astrophysics | Harvard \& Smithsonian, Cambridge, MA, 02138, USA \\ (Received ...; Revised ...; Accepted ...) \\ Submitted to ApJ
}

\begin{abstract}
Energetic particles spectra at interplanetary shocks often exhibit a power law within a narrow momentum range softening at higher energy. We introduce a transport equation accounting for particle acceleration and escape with diffusion contributed by self-generated turbulence close to the shock and by pre-existing turbulence far upstream. The upstream particle intensity steepens within one diffusion length from the shock as compared with diffusive shock acceleration rollover. The momentum spectrum, controlled by macroscopic parameters such as shock compression, speed, far upstream diffusion coefficient and escape time at the shock, can be reduced to a log-parabola and also to a broken power law. In the case of upstream uniform diffusion coefficient, the largely used power law/exponential cut off solution is retrieved.
\end{abstract}

\section{INTRODUCTION}

The diffusion of charged particles at shock waves has been regarded for decades as the major process forging the acceleration of energetic particles (e.g., Drury 1983), with a resulting power-law momentum spectrum (Axford et al. 1977; Bell 1978; Blandford \& Ostriker 1978; Krymskii 1977). A commonplace steepening from single power-law over at least 2 decades in particle momentum is routinely measured in-situ by monitoring suprathermal particles during the arrival of interplanetary shocks to detectors at multiple locations at $1 \mathrm{AU}$ (Lario et al. 2019), or in the time-integrated fluence spectra of energetic particles in Ground Level Enhancements (GLEs, Mewaldt et al. 2012) or in astrophysical observations of radio and $X-/ \gamma$-rays emitted respectively by $\mathrm{GeV}$ and $\mathrm{TeV}$ electrons in supernova remnants ( $\mathrm{Li}$ et al. 2018) or pulsar wind nebulae (Meyer et al. 2010). The question as to what mechanisms govern the unequivocally observed bending spectrum, e.g., escape, transport effects, spherical expansion, shock deceleration and their relative contribution in individual observations remains unanswered; it is also crucial to determine the relative importance of a diffusive shock acceleration (DSA) version that includes escape as compared to, e.g., downstream magnetic reconnection (Zank et al. 2014).

The widespread power law + exponential cut-off spectral fitting model (Ellison \& Ramaty 1985) encapsulates diffusive confinement into the spectral energy break. Double power-laws in solar energetic particle (SEP) spectra have been used to fit time-integrated spectra for the past two decades (e.g. Mason et al. 2002; Mewaldt et al. 2005; Desai et al. 2016). Multi-spacecraft in-situ spectra for GLEs are satisfatorily fit (Mewaldt et al. 2012) by the empirical broken-power-law model introduced by Band et al. (1993) for the astrophysical Gamma-Ray Bursts spectra (e.g., Fraschetti et al. 2006). Cohen \& Mewaldt (2018) found that the 2017 September $10^{\text {th }}$ GLE event spectrum is well fitted by a broken power law. The effect of a finite spatial region in the low corona on the particle acceleration at shocks driven by Coronal Mass Ejections (CME) was investigated (Schwadron et al. 2015) by solving a transport equation that accounts for catastrophic losses, i.e., particle escape from the region where DSA applies, and a broken power-law solution was found. Li \& Lee (2015) have shown that the Parker equation with power-law injected near the Sun admits a double-power-law as a solution at a distance of $1 \mathrm{AU}$ if an energy dependence of the scattering mean free path and adiabatic deceleration in the radially divergent solar wind (SW) are included; however, such study neglects the perpendicular diffusion, that was shown to contribute to the longitudinal spread in large gradual SEP events (Dresing et al. 2012; Dröge et al. 2016, and references therein) and to drifts (Fraschetti \& Jokipii 2011; Dalla et al. 2013). From three IMP8 (Interplanetary Monitory Platform 8) events occurred on September $19^{\text {th }}$, November $22^{\text {nd }} 1977$ and March $1^{\text {st }}$ 1979, Zhao et al. (2017) assessed that

Corresponding author: Federico Fraschetti

ffrasche@1pl.arizona.edu 
the transport across the interplanetary turbulence plays a relevant role, in comparison with the acceleration, in the break of the energetic particles spectra. Bruno et al. (2018) have shown that the $\sim 80 \mathrm{MeV}-f e w \mathrm{GeV}$ spectra of ions collected by PAMELA (Payload for Antimatter Matter Exploration and Light-nuclei Astrophysics) for SEP events are satisfactorily fitted with a power law + exponential rollover. An escape-free model by Drury (2011) yields a spectral break from the combined effect of 1D radial shock expansion and decrease of the acceleration time scale by parametrizing particle injection into DSA. The semi-analytic solution of a model for acceleration and trapping of energetic ions at parallel shocks propagating between 5.8 and 60 solar radii is well fitted with a power law + exponential cutoff (Vainio et al. 2014). Time-integrated broken power law spectra, by implementing the nested shell blast wave approach (Zank et al. 2000; Li et al. 2003; Rice et al. 2003), are supported by ACE and Ulysses data (Verkhoglyadova et al. 2009). Recently, Malkov \& Aharonian (2019) presented a time-dependent model for the escape-free energetic particles intensity across shocks in 3D large-scale spherical geometry expanding in a homogeneous magnetic field and analytically found a spectral steepening. Penetration of astrophysical shocks into neutral-rich molecular clouds was found to steepen the spectrum due to ion-neutral wave-damping (Malkov et al. 2011). Spectra of spike events at interplanetary shocks (Lario et al. 2003) are found numerically to deviate from power law (Fraschetti \& Giacalone 2015) and can be fitted with a particular form of non-power law spectrum, i.e., Weibull function, assuming a time-dependent leaky-box model with a second-order Fermi acceleration (Pallocchia et al. 2017).

In the past two decades, the spectral model of log-parabola was shown to reproduce a broad variety of observations: protons spectra of the 16 GLE events of solar cycle 24 are well fitted by a log-parabola (Zhou et al. 2018). In the astrophysical context, photon spectra in the $X$-ray band of blazars (Massaro et al. 2004) are best-fitted by assuming a log-parabola spectrum of synchrotron-emitting electrons; a significant fraction of photon spectra of extended sources (e.g., supernova remnants) in the third Fermi/Large Area Telescope (LAT) source catalog (Acero et al. 2015) are best-fitted by a log-parabola rather than a single power-law. The photon spectrum of the Crab Nebula in the range $\left(10^{-5}-10^{14}\right) \mathrm{eV}$ (from radio to multi-TeV range), was fitted semi-analytically by a log-parabola spectrum of $\sim \mathrm{TeV}$ electrons (Fraschetti \& Pohl 2017a,b).

The aim of this paper is to formulate a model for particle acceleration and escape in the presence of self-generated turbulence close to the shock and pre-existing turbulence far upstream in order to establish a theoretical foundation of empirical models for energetic particle spectra. A self-consistent theoretical model based on the transport equation coupling the energetic ions and the intensity of upstream ions-generated waves for quasi-parallel magnetic obliquity was built by Lee (1983) and phenomenologically modified to fill the pitch-angle resonance gap by $\mathrm{Ng} \&$ Reames (1994). Kennel et al. (1986) found evidence that a correlation between energetic ions and the upstream wave energy density may exist at interplanetary shocks. Trattner et al. (1994) found such a correlation for 300 energetic ion events at the Earth bow shock. Even for local quasi-perpendicular magnetic obliquity, the upstream medium has been long found to deviate from a laminar structure, both for the Earth bow shock (Fairfield 1974) and for interplanetary shocks, as shown by Wind spacecraft in-situ measurements of large amplitude whistlers precursors (Wilson et al. 2017).

In this paper we present the solution to a new 1-D steady-state transport equation that includes a catastrophic loss term due to particle escape in the presence of self-generated turbulence that matches the pre-existing far upstream turbulence. Steadystate 1-D solutions with a catastrophic loss term incorporating ionization and Coulomb losses, nuclear collisions and adiabatic deceleration behind an expanding shock and with a uniform stationary source were presented early-on by Voelk et al. (1981); a catastrophic loss term describing particle escape from finite extent CME-driven shocks was used to derive broken-power-law momentum spectra by Li et al. (2005).

This paper is organized as follows. In Sect. 2 the relation between the free escape boundary and the energy dependent escape time is illustrated. In Sect. 3 the transport model used herein is outlined with assumed spatial and energy-dependence of the spatial diffusion coefficient. In Sect. 4 we determine analytically the effect on the intensity profile of the particle escape at any energy, not only the highest energy, both from upstream and downstream of the shock for distinct escape times at the shock and distinct particle momenta. In Sect. 5, we determine the effect of the escape on the steady-state 1-D momentum spectrum calculated at the shock, within an ion-inertial length, and compare it with the log-parabola and the broken power law models. In Sect. 6 the results are discussed and Sect. 7 concludes the paper. The Appendix shows that a power law + exponential cut off can be retrieved within the transport model presented herein by assuming a diffusion coefficient uniform throughout except the discontinuity across the shock.

\section{FREE ESCAPE BOUNDARY AND ESCAPE TIME}

In the literature, the upstream particle escape has been customarily modelled by phenomenologically introducing an energyindependent spatial boundary (free-escape boundary, hereafter FEB). Beyond the FEB scattering at any particle energy does not efficiently confine particles that can therefore decouple from the shock and escape with no return to it. Such a spatial cutoff is 
introduced as in the steady-state infinitely planar version of DSA, accelerated particles, once escaped, are ultimately caught up by the shock that is proceeding at constant speed, whereas particles continue to scatter off the far upstream turbulence. Realistic models should include finite shock lifetime, shock deceleration, geometrical effects, large scale spherical shape or small scale corrugation of the shock surface.

The FEB was investigated and numerically implemented by several authors via Monte-Carlo simulations (Jones \& Ellison 1991; Vladimirov et al. 2006), synthesized upstream turbulence test-particle simulations (Giacalone 2005), 1D spherical simulations with cosmic-rays self-generated turbulence (Kang \& Jones 2006), MHD simulations (Reville et al. 2008) with instabilities driven by non-resonant streaming cosmic-rays (Bell 2004); overall, the FEB describes the shock as a leaking finite-size system, beside allowing for a considerable reduction of computational time. However, the location of the FEB, i.e., $x_{F E B}$, is implemented as independent of the particle momentum. As a result, the relative volume density of low- to high-momentum particles, i.e., the momentum spectrum, at every location in steady-state is artificially affected by the choice of $x_{F E B}$, since higher momentum particles would diffuse out to larger distances than low energy particles due to the larger average square displacement, i.e., diffusion coefficient, that allows them to diffuse further away from the shock with respect to lower energy particles and still be able to return to it; such a return is prevented by an energy-independent FEB. Drury (2011) pointed out the dichotomy resulting from introducing an artificial spatial cutoff such as the FEB: models should merge into a unified picture two competing effects, namely the decrease of acceleration efficiency, due for instance to the shock deceleration, and gradual particle escape, occurring at all energies, not only the highest ones. The aim of this paper is to outline a viable merge of the two processes.

A second consequence of the energy-independence of $x_{F E B}$ can be seen as follows. The location $x_{F E B}$, in addition to providing the upstream region of influence of the shock, can be related to a diffusive escape time that can be expressed as $\Delta t(p) \propto$ $x_{F E B}^{2} / \kappa(p)$ where $\kappa(p)$ is the momentum-dependent spatial diffusion coefficient. In the modelling of $i n$-situ intensity profiles, a momentum-independent $x_{F E B}$ is in tension with the measurements, that show that even far upstream the intensity profile at distinct particles energy flattens down to the background level at distinct distances from the shock (e.g., Lario et al. 2019). Thus, a momentum-dependence of $x_{F E B}$, or in other terms a momentum-dependence of $\Delta t(p)$ distinct from the momentum-dependence of $\kappa^{-1}$, ensures a more realistic description of the intensity profiles that allows particles at distinct energy to escape at distinct locations, not at a unique $x_{F E B}$. Such an effect ultimately changes also the upstream steady-state spectrum. We aim at relating spectral signatures of particle escape (softening) with shock properties (e.g., compression, speed) both in single spacecraft spectra and in multi-spacecraft time-integrated fluences (Mewaldt et al. 2012).

From a shock with infinite lifetime and constant speed no particle can disappear as all particles diffusing upstream are eventually caught up; however, neither assumption applies to real shocks. Here we introduce an escape time to account for the two forementioned limitations of the DSA. The particle escape is mimicked here by introducing the escape time scale $T(x, p)$ that depends on both position and momentum. We note that in the transport equation the two separate terms of spatial diffusion and escape (in the form of catastrophic losses) are not redundant as each one carries distinct physical information: upstream of a constant speed shock the only diffusion coefficient, despite dependent on momentum and increasing with the distance from the shock, does not entail that, during the shock lifetime, escaped particles will ever return to the shock. Some of them are scattered back and might return to it and others escape. The herein introduced $T(x, p)$ allows to differentiate those that scatter back and return to the shock, keeping a diffusive scattering at the shock, from those genuinely, i.e., observationally, escaped.

In the downstream plasma, the diffusion competes with both escape and flow advection: at each location $x$, for $T$ longer than the advection time scale, i.e., $x / U$ (where $x$ is the average direction of the shock motion and $U$ the shock speed in the downstream plasma frame), the advection dominates and particles are advected with the flow although some can backscatter and return to the shock (for such a last sub-population, $T$ would be the relevant time scale rather than $x / U$ so that those particles are accounted for with a proper choice of $T>x / U$ ). The opposite case, namely $T<x / U$, describes high energy particles with velocity component along the flow much greater than $U$. As shown in the Sect. 4, the resulting spatial profile differs considerably from the uniform profile predicted by DSA, due to a drop steeper for shorter $T$. In summary, in the 1D case developed below the downstream escape incorporated in $T$ can account both for particles so much faster than the bulk flow speed that diffusion can not efficiently confine them and for backscattering particles able to return to the shock. Geometrical effects such as finite extension or corrugation of the shock surface are not incorporated in $T$ here and require a 2D approach.

\section{OUTLINE OF THE MODEL}

We consider an infinitely planar shock wave. We use the simplifying assumption that the number of particles per unit time undergoing injection and acceleration processes is balanced by the number of particles per unit time escaping the system so that the steady-state condition holds: $\partial f / \partial t=0$, where $f(x, p)$ is the phase-space distribution function for the energetic particles. Realistic models should describe also the time-dependent imbalance between acceleration and escape; for the sake of simplicity, 
this effect is neglected herein. We can cast the 1-D steady-state transport equation, assuming pitch-angle isotropy in the local plasma frame, as:

$$
U \frac{\partial f(x, p)}{\partial x}=\frac{\partial}{\partial x}\left[\kappa(x, p) \frac{\partial}{\partial x} f(x, p)\right]+\frac{1}{3}\left(\frac{d U}{d x}\right) p \frac{\partial f(x, p)}{\partial p}+S(x, p)-\frac{f(x, p)}{T(x, p)},
$$

where $S(x, p)$ is the source term and the flow speed in the shock frame is uniform both upstream and downstream and discontinuous at the shock on the energetic ions inertial scale:

$$
U= \begin{cases}U_{1} & \text { if } x<0, \text { upstream } \\ U_{2} & \text { if } x>0, \text { downstream }\end{cases}
$$

During the last four decades, a vast literature explored the non-linear mechanisms (e.g., Malkov \& Drury 2001) exciting the turbulence upstream of shock waves, e.g. resonant instability by the energetic ions diffusing upstream. The density of the scattering centers decreases linearly and $\kappa$ increases linearly upstream for an Alfvénic self-generated turbulence (Bell 1978); for instance, the power spectrum of Alfénic fluctuations for high Mach number (supernova remnant) shocks was shown numerically to decrease for each wavenumber over a range of $\sim 4$ decades as the upstream distance from the shock increases (Brose et al. 2016). Far upstream, the pre-existing interplanetary/interstellar medium turbulence (Armstrong et al. 1995) provides a cutoff out at a certain distance from the shock, i.e., $x=\Lambda_{1}$, where the self-generated turbulence becomes negligible and $\kappa$ reaches a uniform value, rather than increasing indefinitely (see Fig. 1). The value of $\Lambda_{1}=10^{11} \mathrm{~cm}$ is consistent with the extension of the suprathermal $(1-30 \mathrm{keV})$ proton foreshocks measured, e.g., in STEREO (Solar TErrestrial RElations Observatory) interplanetary shocks (between 0.02 and 0.1 AU, Kajdič et al. 2012).

The instability driven by the upstream current of non-resonant energetic ions (Bell 2004) generates distinct, i.e., non-linear and non-Alfvénic, fluctuations that contributes to reducing the $\kappa$ of the lower-energy ions. The power spectrum of such fluctuations for high Mach number shocks was shown by Monte Carlo simulations to decrease for each wavenumber over a range of $\sim 8$ decades as the upstream distance from the shock increases (Bykov et al. 2014). However, in the absence of a simple functional dependence of $\kappa$ on position inferred from in-situ measurements or simulations, we assume a linear increase of the upstream $\kappa$ (Bell 1978).

The downstream turbulence is advected with the fluid with a typical length-scale much greater than the upstream diffusion scale, with a corresponding weak spatial dependence of $\kappa$; thus, $\kappa$ is assumed to be uniform here. Upstream large-scale density inhomogeneities lead to exponentially rapid amplification of the downstream magnetic field (Giacalone \& Jokipii 2007; Fraschetti 2013, 2014); for fluctuations length scale as large as the gyroscale of $\lesssim$ GeV protons at 1 AU (namely 0.07 AU), such a generated turbulence might introduce a spatial dependence of the downstream $\kappa$. However, the field amplification scales with the Alfvén Mach number $M_{A}$ (Fraschetti 2013) and is modest at the $M_{A}<10$ interplanetary shocks ${ }^{1}$. In addition, the specific location of the most efficient particle acceleration process (whether at the shock or downstream) is still topic of debate. Transport models have interpreted the measured rise of energetic particle intensity downstream of interplanetary shocks (Lario et al. 2003) as footprint of an additional source of acceleration therein due to magnetic reconnection (Zank et al. 2014, 2015). Also, measurements of Voyagers in the heliosheath, between the termination shock and the heliopause, show a rise of anomalous cosmic rays $(\sim 10-100$ $\mathrm{keV}$ particles) with a peak at a distance of $\sim 1 \mathrm{AU}$ from the shock (Zhao et al. 2019), hence questioning the relative role of the shock transition layer in the acceleration. Equation 1 does not include a downstream source of acceleration.

We simplify the problem by assuming that $\kappa$ is separable in the following hybrid dependence (see Fig. 1):

$$
\kappa(x, p)= \begin{cases}\kappa_{1}(p) \frac{|x-\epsilon|}{\left|\Lambda_{1}\right|} & \text { if } x<0 \text { and }|x|<\left|\Lambda_{1}\right|, \text { upstream } \\ \kappa_{1}(p) & \text { if } x<0 \text { and }|x|>\left|\Lambda_{1}\right|, \text { far upstream } \\ \kappa_{2}(p) & \text { if } x>0, \text { downstream }\end{cases}
$$

where $\kappa_{i}(p)$ depends only on momentum and $|x-\epsilon| /\left|\Lambda_{1}\right|$ only on space, $i=1,2$ indicates respectively upstream and downstream, $\epsilon=d_{i} \simeq 10^{7} \mathrm{~cm}$ is the ion inertial length. Here $\kappa \rightarrow \kappa_{1}(p)$ for $x \rightarrow \Lambda_{1}$ is the far upstream diffusion coefficient in the preexisting turbulence. We assume also the separability of $T$; the upstream $T$ is expected to decrease with the distance from the shock due to the smaller density of scattering centers far from the shock, and higher close to it, whereas the downstream $T$ is

\footnotetext{
${ }^{1}$ In contrast, at supernova remnant shocks the amplified turbulent field downstream is stronger $\left(M_{A} \simeq 100-1,000\right)$, the gyro-scale of the highest energy protons $\left(\sim 10^{14}-10^{15} \mathrm{eV}\right)$ is comparable with the scale of the inhomogeneities and the downstream $\kappa$ might be spatially dependent.
} 


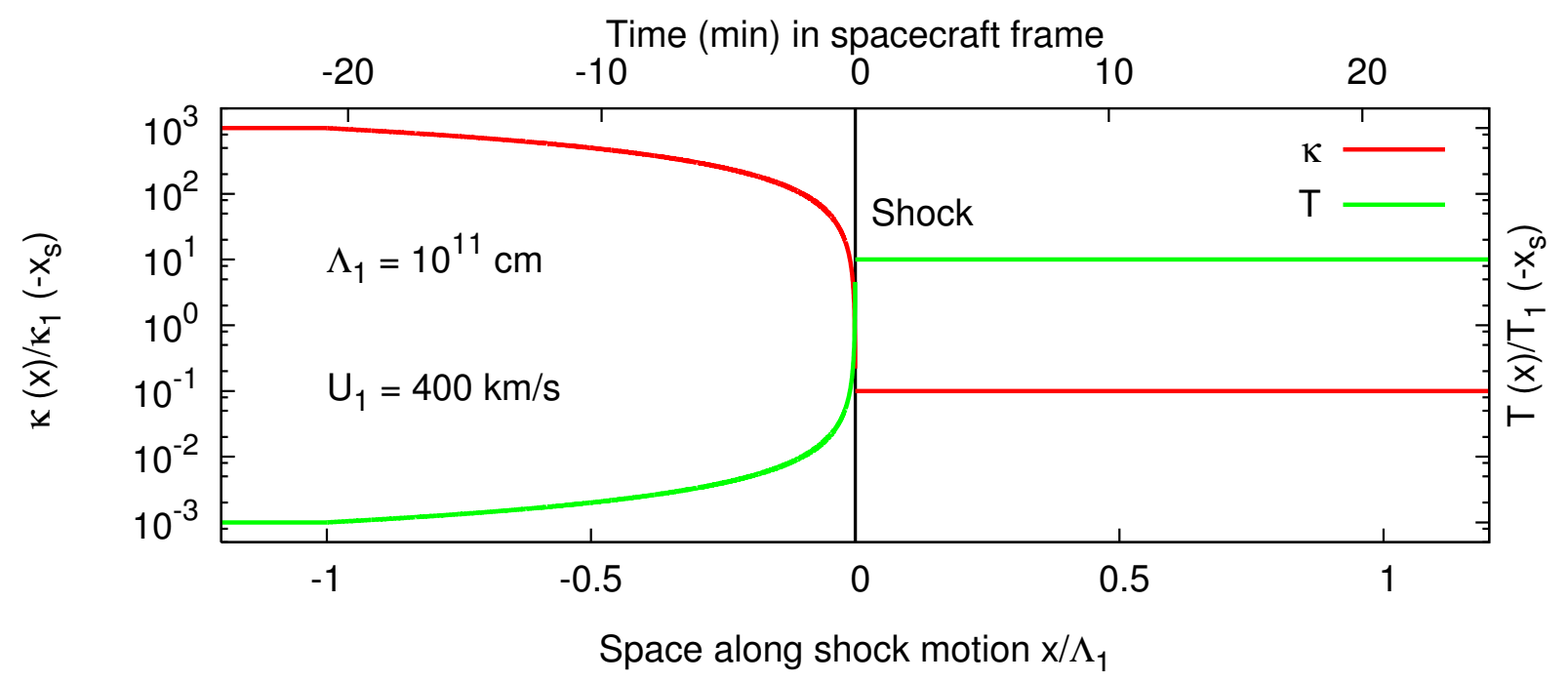

Figure 1. Spatial profiles of $\kappa$ and $T$ at a given momentum in units of respective values upstream at distance $\left|-x_{s}\right|=10 d_{i}$ from the shock, where $d_{i} \simeq 10^{7} \mathrm{~cm}$ is the ion inertial length. The upper $x$-axis indicates the time in the spacecraft frame using $U_{1}=400 \mathrm{~km} / \mathrm{s}$ at a SW speed $400 \mathrm{~km} / \mathrm{s}$.

expected to be uniform, mimicking the $\kappa$ dependence. Thus,

$$
T(x, p)= \begin{cases}T_{1}(p)\left|\Lambda_{1}\right| & \text { if } x<0 \text { and }|x|<\left|\Lambda_{1}\right|, \text { upstream } \\ T_{1}(p) & \text { if } x<0 \text { and }|x|>\left|\Lambda_{1}\right|, \text { far upstream } \\ T_{2}(p) & \text { if } x>0, \text { downstream. }\end{cases}
$$

The equations in this Sect. can be compared with the FEB approach that assumes that $f\left(x_{F E B}\right)=0$ and that the diffusive flux is described by $\kappa(x) \partial f /\left.\partial x\right|_{x=x_{F E B}}$. The solution presented below uses the fact that, from Eqs. 2, 3, the product $\kappa_{i} T_{i}$ is independent of space, although the upstream value $\kappa_{1} T_{1}$ might differ from the downstream value $\kappa_{2} T_{2}$.

\section{ENERGETIC PARTICLES INTENSITY PROFILE}

\subsection{Upstream intensity profile}

The transport Eq. 1 with the assumptions outlined in Sect. 3 can be solved analytically in position space. The general solution upstream ( $U=U_{1}$ so that $d U / d x=0$ and far from the source $\left.S(x, p)=0\right)$ is found by solving

$$
U_{1} \frac{\partial f(x, p)}{\partial x}=\frac{\partial}{\partial x}\left[\kappa_{1}(x, p) \frac{\partial}{\partial x} f(x, p)\right]-\frac{f(x, p)}{T(x, p)} .
$$

We factorize $f$ as $f(x)=u(x) v(x)$, where $u(x)$ and $v(x)$ are solutions respectively to the equations

$$
\frac{\partial^{2} u}{\partial x^{2}}+\left[-\frac{1}{\kappa_{1}(x, p) T_{1}(x, p)}-\frac{4 \nu_{1}^{2}-1}{4 x^{2}}\right] u=0, \quad \frac{\partial^{2} v}{\partial x^{2}}+\frac{1}{2}\left[\frac{\partial a}{\partial x}-\frac{a^{2}}{2}\right] v=0,
$$

where $\nu_{1}(p)=\left|\Lambda_{1}\right| U_{1} / 2 \kappa_{1}(p)$ and $a=\left(\partial \kappa_{1} / \partial x-U_{1}\right) / \kappa_{1}$. The solution to the $u$-equation is a linear combination of Bessel functions (see Gradshteyn et al. 2007, Eq. 8.491). The phase-space distribution function $f(x, p)$ in the upstream can be recast as

$$
f(x, p)=\underbrace{\left[C_{1} J_{\nu}\left(\frac{i|x-\epsilon|}{L_{1}(p)}\right)+C_{2} Y_{\nu}\left(\frac{i|x-\epsilon|}{L_{1}(p)}\right)\right]}_{\mathrm{u}(\mathrm{x})} \underbrace{\exp \left(-\int_{x}^{0} \frac{U\left(x^{\prime}\right)}{2 \kappa\left(x^{\prime}, p\right)} d x^{\prime}\right)}_{\mathrm{v}(\mathrm{x})}
$$

where $L_{1}(p) \equiv \sqrt{\kappa_{1}(x, p) T_{1}(x, p)}=\sqrt{\kappa_{1}(p) T_{1}(p)}$ is defined here as the escape length, independent of the distance from the shock, $J_{\nu}, Y_{\nu}$ are Bessel functions of first kind and second kind, respectively, $i$ is the imaginary unit and $C_{1}, C_{2}$ are constants to be determined by boundary conditions; the last exponential factor is reminiscent of the upstream steady-state 1D solution for an infinitely planar shock in the case of no escape (DSA). Since the spatial dependencies of $\kappa$ and $T$ are expected to be reciprocal or 
nearly reciprocal, a choice of such dependencies different from Eqs. 2 and 3 might introduce at most a weak spatial dependence of $L_{1}(p)$.

By imposing that $f(x, p)$ is not exponentially divergent far upstream $(f(x, p) \not \rightarrow \infty$ as $x \rightarrow-\infty)$ leads to the relation $C_{2}=i C_{1}$; this is found by using the asymptotic expressions for $J_{\nu}, Y_{\nu}$ (see Gradshteyn et al. 2007, Eq.s 8.451.1 - 8.451.2) and taking only the term $k=0$ of the $k$-expansion.

The complex-valued constant $C_{1}$ is determined by imposing $f(x, p)=f_{0}(p)$ at the shock $(x=0)$. We use the series representations of $J_{\nu}, Y_{\nu}$ (see Gradshteyn et al. 2007, Eq.s $8.440-8.443-8.451 .2$ ) and take only the term $k=0$ of the expansion since $x \simeq 0$.

The exact upstream solution to Eq. 1, where the integration constants are calculated using the boundary conditions and approximations described above, can then be recast as

$$
f(x, p)=f_{0}(p) \Gamma\left(1-\nu_{1}\right)\left(\frac{|x-\epsilon|}{2 L_{1}(p)}\right)^{\nu_{1}} \mathcal{R}\left[-\mathrm{e}^{-i \pi \nu_{1}} I_{\nu_{1}}\left(-\frac{|x-\epsilon|}{L_{1}(p)}\right)+I_{-\nu_{1}}\left(\frac{|x-\epsilon|}{L_{1}(p)}\right)\right]
$$

where $I_{\nu_{1}}(z)$ is the modified Bessel functions of imaginary argument (see Gradshteyn et al. 2007, Eq. 8.406.1), $\Gamma(z)$ is the gamma function and $\mathcal{R}[$.$] indicates the real part of [.]. The relevant limits of Eq. 6$ are analysed below.

The asymptotic limit of Eq. 6 for $|x-\epsilon| \gg L_{1}(p)$, by using the asymptotic expression of $I_{\nu}(z)$ in Gradshteyn et al. (2007), Eq. 8.451.5, and taking only the term $k=0$, leads to the following upstream profile

$$
f(x, p) \rightarrow f_{0}(p) \frac{\Gamma\left(1-\nu_{1}\right)}{\sqrt{\pi}} \sin \left(\pi \nu_{1}\right)\left(\frac{|x-\epsilon|}{2 L_{1}(p)}\right)^{\nu_{1}-1 / 2} \exp \left(-\frac{|x-\epsilon|}{L_{1}(p)}\right), \text { upstream. }
$$

We note that the $\Lambda_{1}$-dependence of the upstream profile $f(x, p)$ is implicit in $\nu_{1}(p)$. The spatial profile in Eq. 7 has to be compared with the steady state 1D test-particle solution of DSA with no upstream escape. We readily note that for large distance from the shock, i.e., limit $|x| \gg L_{1}$ (and $\left.|x|<\left|\Lambda_{1}\right|\right), f$ is exponentially suppressed with a roll-over scale $L_{1}(p)$, that replaces the roll-over scale in the case of no escape (DSA), i.e., $\sim \kappa_{1}(\epsilon, p) / U_{1}$. Thus, particles do not disappear and still spread out to an infinite distance as in DSA, but with an exponentially small amplitude.

The DSA upstream profile is recovered if, in the surrounding of a certain far upstream location $|\bar{x}| \gg\left|x_{s}\right|$, the escape time becomes comparable with the acceleration time:

$$
T_{1}(\bar{x}, p) \simeq \frac{\kappa_{1}(\bar{x}, p)}{U_{1}^{2}},
$$

where the right-hand side of Eq. 8 is a crude estimate of the acceleration time (Axford 1981; Forman \& Drury 1983; Drury 1983). This limit can be clarified as follows. If the condition in Eq. 8, equivalent to $\kappa_{1}(\bar{x}, p) / U_{1} \simeq L_{1}(p)$, is satisfied, the last exponential factor of Eq. 7 becomes $\exp \left[-U_{1}|\bar{x}-\epsilon| / \kappa_{1}(\bar{x}, p)\right]$, i.e., the profile in the case of no-escape: $\exp \left(-\int_{x}^{0} d x^{\prime} U_{1} / \kappa_{1}\left(x^{\prime}, p\right) \simeq\right.$ $-U_{1} \Delta x / \kappa_{1}(\bar{x}, p)$, where $\kappa_{1}(\bar{x}, p)$ is interpreted as an average of $\kappa_{1}$ within the upstream interval $(x, 0)$. The only additional spatially-dependent factor in Eq. 7, i.e., $\left(|x-\epsilon| / 2 L_{1}(p)\right)^{\nu_{1}-1 / 2}$, absent in the no-escape solution, tends to zero for large $|x|$ as $\nu_{1}<1 / 2$ for the particle energy of interest herein:

$$
\nu_{1}\left(p_{0}\right)=0.1 \frac{\left|\Lambda_{1}\right|}{10^{11} \mathrm{~cm}} \frac{U_{1}}{200 \mathrm{~km} / \mathrm{s}}\left(\frac{\bar{\kappa}_{1}}{10^{19} \mathrm{~cm}^{2} / \mathrm{s}}\right)^{-1} ;
$$

where we have introduced the normalization constant $\bar{\kappa}_{1}=\kappa_{1}\left(\Lambda_{1}, p_{0}\right)$; the typical value of $\kappa_{1}\left(\Lambda_{1}, p_{0}\right) \simeq 10^{19} \mathrm{~cm}^{2} / \mathrm{s}$ for $p_{0}$ corresponding to $\sim 100 \mathrm{keV}$ protons is consistent with the mean free path measured across the interplanetary medium (Palmer 1982). Equation 9 shows that even for very fast shocks and small particle momentum (small $\kappa$ ), the inequality $\nu_{1}<1 / 2$ is likely to be satisfied. We note that the assumptions herein lead to a ratio of acceleration-to-escape time scale, $\kappa_{1}(x, p) / U_{1}^{2} T_{1}(x, p)$, increasing quadratically with the distance from the shock, as $\kappa_{1} \propto x$ and $T_{1} \propto 1 / x$ (see Eqs. 2, 3); thus, the likelihood of upstream escape is continuously varying as particles progress upstream rather than being governed by an artificial switch operating only at $x=x_{F E B}$.

Illustrative monochromatic $\left(p=p_{0}\right)$ intensity profiles are shown in Fig. 2, upper panel, for $T_{1}\left(-x_{s}\right)=400 \mathrm{sec}$ upstream at a distance from the shock $\left|-x_{s}\right|=10 d_{i}$ and distinct ratios $T_{2} / T_{1}\left(-x_{s}\right)$. The diffusion coefficient for $\sim 100 \mathrm{keV}$ protons at the shocks is chosen as $\kappa_{1}\left(-x_{s}, p_{0}\right)=10^{16} \mathrm{~cm}^{2} / \mathrm{s}$; smaller values of $\kappa_{1}$ (leading to higher acceleration rates, Jokipii 1982, 1987) are not ruled out at local high obliquity shocks. The DSA profile is drawn for comparison (in cyan) with an upstream $\kappa_{1}(\bar{x}, p)=\kappa_{1}^{D S A}=10^{17} \mathrm{~cm}^{2} / \mathrm{s}$ so that the diffusion scale is $\kappa_{1}^{D S A} / U_{1}$ equals the escape length $L_{1}\left(p_{0}\right)=2 \times 10^{9} \mathrm{~cm}$. The 
value of $\kappa_{1}^{D S A}$ is consistent with the recent determination by Kis et al. (2018) of the e-folding distance ahead of quasi-parallel obliquity regions of the Earth bow shock for $\sim 10-40 \mathrm{keV}$ protons. The insert in the upper panel in Fig. 2 shows that the upstream escape depletes the particle intensity very close to the shock, within $L_{1}\left(p_{0}\right)$, to $\sim 20 \%$ of the DSA prediction due to the factor $\left(|x-\epsilon| / 2 L_{1}(p)\right)^{\nu_{1}-1 / 2}$ (see Eq. 7); far upstream the intensity dilutes asymptotically with comparable slope, in logarithmic scale, as DSA due to $\kappa_{1}^{D S A} / U_{1}=L_{1}\left(p_{0}\right)$. An analysis (Kis et al. 2004) of multi-spacecraft $10-32 \mathrm{keV}$ ion events at the Earth bow shock led to an estimate of the roll-over upstream distance shorter than the statistical expectation (Trattner et al. 1994). The profile determined in Eq. 7 suggests an efficient escape as explanation of the short roll-over distance, in alternative to the explanation based on a large SW velocity (Kis et al. 2004).

The lower panel in Fig. 2 shows that a long escape time $\left(T_{1}\left(-x_{s}\right)=8,000 \mathrm{sec}\right.$, blue line) leads to a rapid drop within the DSA diffusion length $\left(\kappa_{1}^{D S A} / U_{1}\right.$, corresponding to $\sim 0.37 \mathrm{~min}$ in spacecraft frame); the shallower decrease of the blue curve far upstream, that leads to the crossing of the blue curve with the DSA curve, results from the increase of $\kappa_{1}$ with distance from the shock that increases the effective diffusion length far from the shock. Equation 8 , equivalent to $\kappa_{1}(\bar{x}, p) / U_{1} \simeq L_{1}(p)$, shows that if $T_{1}(p)$ is smaller than the acceleration time scale at $x=\bar{x}, L_{1}$ is the length scale of the roll-over profile; if $T_{1}(p)$ becomes comparable to the acceleration time scale, the escape is not a dominant process and the profile is governed by acceleration and diffusion, as in the case of DSA. We note that if $T$ and $\kappa$ have different momentum dependence, the threshold in Eq. 8 is met at distinct locations for distinct momenta. Thus, Eq. 8 illustrates quantitatively the balance between escape and acceleration.

We note that the condition of no-divergence far upstream imposed on $f(x, p)$ is consistent with an escape time scale shorter than the acceleration time scale at large distance from the shock, thereby efficient escape from the shock over a broad range of distances from the shock for distinct particle energy. From the assumed dependencies in Eq. 2, it holds (for $\left|-x_{s}\right|<\left|\Lambda_{1}\right|$ )

$$
\exp \left(-\int_{x}^{0} \frac{U\left(x^{\prime}\right)}{2 \kappa\left(x^{\prime}, p\right)} d x^{\prime}\right)=\left(\frac{|x-\epsilon|}{\epsilon}\right)^{\nu_{1}}
$$

By using the asymptotic expressions for the Bessel functions (see Gradshteyn et al. 2007, Eq.s 8.451.1 - 8.451.2), we can recast the spatially dependent factors of $f(x, p)$ in Eq. 7 as

$$
\left(\frac{|x-\epsilon|}{\epsilon}\right)^{\nu_{1}} \exp \left(-\frac{|x-\epsilon|}{L_{1}(p)}\right)=\exp \left(\nu_{1} \ln \frac{|x-\epsilon|}{\epsilon}-\frac{|x-\epsilon|}{L_{1}(p)}\right) .
$$

Although the left hand side in Eq. 10 is clearly exponentially suppressed for any value of the parameters $\nu_{1}, \kappa_{1}(p)$ and $T_{1}(p)$, it is useful to note that the condition

$$
\nu_{1} \ln \frac{|x-\epsilon|}{\epsilon}-\frac{|x-\epsilon|}{L_{1}(p)} \lesssim 0,
$$

that is necessary to prevent exponential divergence, implies far upstream

$$
T_{1}(p) \lesssim \frac{\kappa_{1}(p)}{U_{1}^{2}} .
$$

Equation 11 links the mathematical condition of no-divergence of the solution with the fact that acceleration cannot balance escape and particles at any momentum are allowed to escape, a different times $T_{1}(p)$ (cfr. Eq. 8), again emphasizing that the approach presented herein describes the continuous process of escape at any particle energy.

We note that, although $L_{1}$ does not depend explicitly on the advection speed $U_{1}$ unlike the diffusion scale, the escape in this $1 \mathrm{D}$ case depends on the balance between advection and diffusive confinement (confinement is assumed to be infinitely efficient at any particle energy in the case of no escape within a given FEB), as in the DSA. In the presence of 2D shock the geometry is expected to contribute the particles confinement.

Finally, from a mathematical viewpoint, at large distance from the shock $f$ has to tend to zero and cannot be a finite constant since the only uniform solution of Eq. 1 is the identically zero distribution. This is at odds with the case of no escape (i.e., $f(x, p) / T \sim 0$ in Eq. 1): a non-vanishing constant depending only on momentum is the asymptotic upstream solution.

\subsection{Downstream intensity profile}

In the downstream region ( $U=U_{2}$ so that $d U / d x=0$ and far from the source $\left.S(x, p)=0\right)$, we impose continuity with the upstream solution, i.e., $f(0, p)=f_{0}(p)$; then, taking the limit for $x \rightarrow+\infty$ and discarding the exponentially divergent solution yields

$$
f(x, p)=f_{0}(p) \exp \left[\left(\frac{U_{2}}{2 \kappa_{2}(p)}-\frac{1}{2} \sqrt{\left(\frac{U_{2}}{\kappa_{2}(p)}\right)^{2}+\frac{4}{L_{2}^{2}(p)}}\right) x\right], \text { downstream }
$$


where $L_{2}(p) \equiv \sqrt{\kappa_{2}(p) T_{2}(p)}$ is the downstream escape-length. In the limit of no-escape $\left(T_{2}(p) \gg \kappa_{2}(p) / U_{2}^{2}\right.$ or, equivalently, $\left.L_{2}(p) \gg \kappa_{2}(p) / U_{2}\right)$, the downstream profile tends to the uniform limit $f_{0}(p)$, recovering the DSA solution. Equations 7 and 12 show that the profiles depend on the ratio $\kappa(p) / U L(p)$ (upstream coinciding with the ratio of the diffusion length to the escape length): if $T(p)$ is very large the DSA solution is retrieved.

In the downstream region, Fig. 2 (upper panel) shows that large escape times $\left(T_{2}>2,000 \mathrm{sec}\right)$ as compared to the advection time ( $\sim$ a few minutes) leads the profile to the DSA flat shape, as the escape becomes irrelevant and the profile is advectiondominated. As $T_{2}$ is shortened and becomes comparable to the advection time, the profile drops more and more steeply behind the shock due to particles moving downstream away from the shock and faster than the advected flow.

Figure 2, upper panel, shows intensity profiles for fixed $\kappa_{1}\left(-x_{s}, p_{0}\right) / \kappa_{2}\left(p_{0}\right)=10$ and distinct values of $T_{2}\left(p_{0}\right) / T_{1}\left(-x_{s}, p_{0}\right)$ within the shock layer. The case $\kappa_{1}\left(-x_{s}, p_{0}\right) / \kappa_{2}\left(p_{0}\right)=T_{2}\left(p_{0}\right) / T_{1}\left(-x_{s}, p_{0}\right)=10$, i.e., $L_{1}\left(p_{0}\right)=L_{2}\left(p_{0}\right)$, is shown by the upstream and downstream red curves. For this case the upstream curve falls steeper than the DSA curve (cyan curve in the insert) close to the shock but reaches the same asymptotic slope; the downstream profile is steeply declining, in contrast with the DSA case. Only increasing $T_{2} / T_{1}$ at fixed $\kappa_{1} / \kappa_{2}\left(L_{1}<L_{2}\right)$ leads to a flatter downstream profile closer to the DSA prediction. Thus, a comparison with DSA only, suggests $L_{1}<L_{2}$; however, measured upstream profiles steeper than DSA suggest that the case $L_{1}>L_{2}$ can be realised. High time-resolution spacecraft data at terrestrial bow shock or interplanetary shocks can help investigate and constrain this model. Figure 2, upper panel, shows roll-over timescale of $\sim 1$-minute; the high cadence of, e.g. the Magnetospheric Multiscale (MMS) mission, Hot Plasma and Energetic Particles Detector (Cohen et al. 2019), allows an accurate estimate of the shock parameters and of energetic particles profile within such a time interval.

\subsection{Profile for distinct particle momentum}

Figure 3 shows the dependence of the intensity profile on $p$ under the assumption of a power-law $p$-dependence of $\kappa$ and $T$ :

$$
\kappa_{i}(p)=\bar{\kappa}_{i}\left(p / p_{0}\right)^{\delta_{i}}, \quad T_{i}(p)=\bar{T}_{i}\left(p / p_{0}\right)^{-\gamma_{i}}, \quad \delta_{1}>\gamma_{1}
$$

where $\bar{\kappa}_{1}$ is defined below Eq. 9, $\bar{\kappa}_{2}=\kappa\left(p_{0}\right), \bar{T}_{1}=T_{1}\left(\Lambda_{1}, p_{0}\right), \bar{T}_{2}=T_{2}\left(p_{0}\right)$ and power-law indexes $\delta_{i}>0, \gamma_{i}>0$; the condition $\delta_{1}>\gamma_{1}$ is motivated below. The value of $\delta_{i}$ depends on the particular turbulence assumed in the upstream medium ( $\delta_{i}=4 / 3$ in the 3D isotropic turbulence and $\delta_{i}=1$ in the Bohm diffusion limit). The value of $\gamma_{i}$ is relatively unconstrained. In this figure we keep for the sake of simplicity $\delta_{1}=\delta_{2}$ and $\gamma_{1}=\gamma_{2}$, neglecting the effects of compression or magnetic field amplification at the shock. For two distinct values of momentum $\left(p_{0}\right.$ and $\left.2 p_{0}\right)$ and $T_{1}\left(-x_{s}, p_{0}\right)=100 \mathrm{sec}$, Fig. 3 compares the intensity profiles with the respective DSA prediction such that $\kappa_{1}^{D S A}\left(p_{0}\right) / U_{1}=L_{1}\left(p_{0}\right)=10^{9} \mathrm{~cm}$, assuming the same $\delta_{1}$. Larger momenta lead to larger diffusion length $\left(\kappa_{1}^{D S A} / U_{1}\right)$; likewise, the condition $\delta>\gamma$ leads to an increasing $L_{1}(p) \propto\left(p / p_{0}\right)^{\left(\delta_{1}-\gamma_{1}\right) / 2}$ with momentum (as in Fig. 3), thereby explaining the crossing of the thick red and green curves upstream: the factor $\left(\frac{|x-\epsilon|}{2 L_{1}(p)}\right)^{\nu_{1}-1 / 2}$ in Eq. 7 steepens the profile close to the shock at larger $p$ due to the $\nu_{1}(p)$ dependence (see Eq.9) but $L_{1}(p)$ is also larger for larger $p$ thereby diluting the profile far upstream. The profiles in Fig. 3 are generally in agreement with the spacecraft measurements that higher energy particles diffuse out to larger distances from the shock than lower energy particles. Thus, the case $\delta<\gamma\left(\right.$ leading to $L_{1}(p)$ also smaller for larger $p$ ) is ruled out by in-situ measurements. In addition, we note that the assumptions in Eqs. 13 also imply that the ratio $\kappa_{1}(x, p) / U_{1}^{2} T_{1}(x, p)$ increases with a power-law index in momentum $\delta_{1}+\gamma_{1}>1$ (see Eq.13), satisfied for any value $\gamma$ for the turbulence models mentioned above ( $\left.\delta \geq 1\right)$, consistently with the expectation that for higher $p$ the escape is favoured over the acceleration.

\section{MOMENTUM SPECTRUM}

From the continuity of $f(x, p)$ across the shock, the momentum spectrum at the shock $f_{0}(p)=f(p)$ can be derived following the usual textbook procedure in the case of DSA. The conservation of the number of particles flowing along the $x$-direction across the shock, i.e., $\int_{-\epsilon}^{+\epsilon} d x U \partial f / \partial x=0$, applied to Eq. 1 reads:

$$
\left.\left[\kappa(p) \frac{\partial f(x, p)}{\partial x}+\frac{1}{3} U p \frac{\partial f(x, p)}{\partial p}\right]\right|_{-\epsilon} ^{+\epsilon}+\int_{-\epsilon}^{+\epsilon} d x\left[S(x, p)-\frac{f(x, p)}{T(p)}\right]=0 .
$$

From the upstream exact solution for a spatially varying $\kappa_{1}(x, p)$ in Eq. 6 and the recursion formula for the derivative of $I_{\nu}(z)$ (Gradshteyn et al. 2007, see Eq. 8.486.1), it holds

$$
\left.\frac{\partial f(x, p)}{\partial x}\right|_{-\epsilon} \simeq f_{0}(p) \frac{\Gamma\left(1-\nu_{1}(p)\right)}{\Gamma\left(1+\nu_{1}(p)\right)} \frac{\nu_{1}(p)}{2 L_{1}(p)}\left(\frac{\epsilon}{2 L_{1}(p)}\right)^{2 \nu_{1}(p)-1}
$$



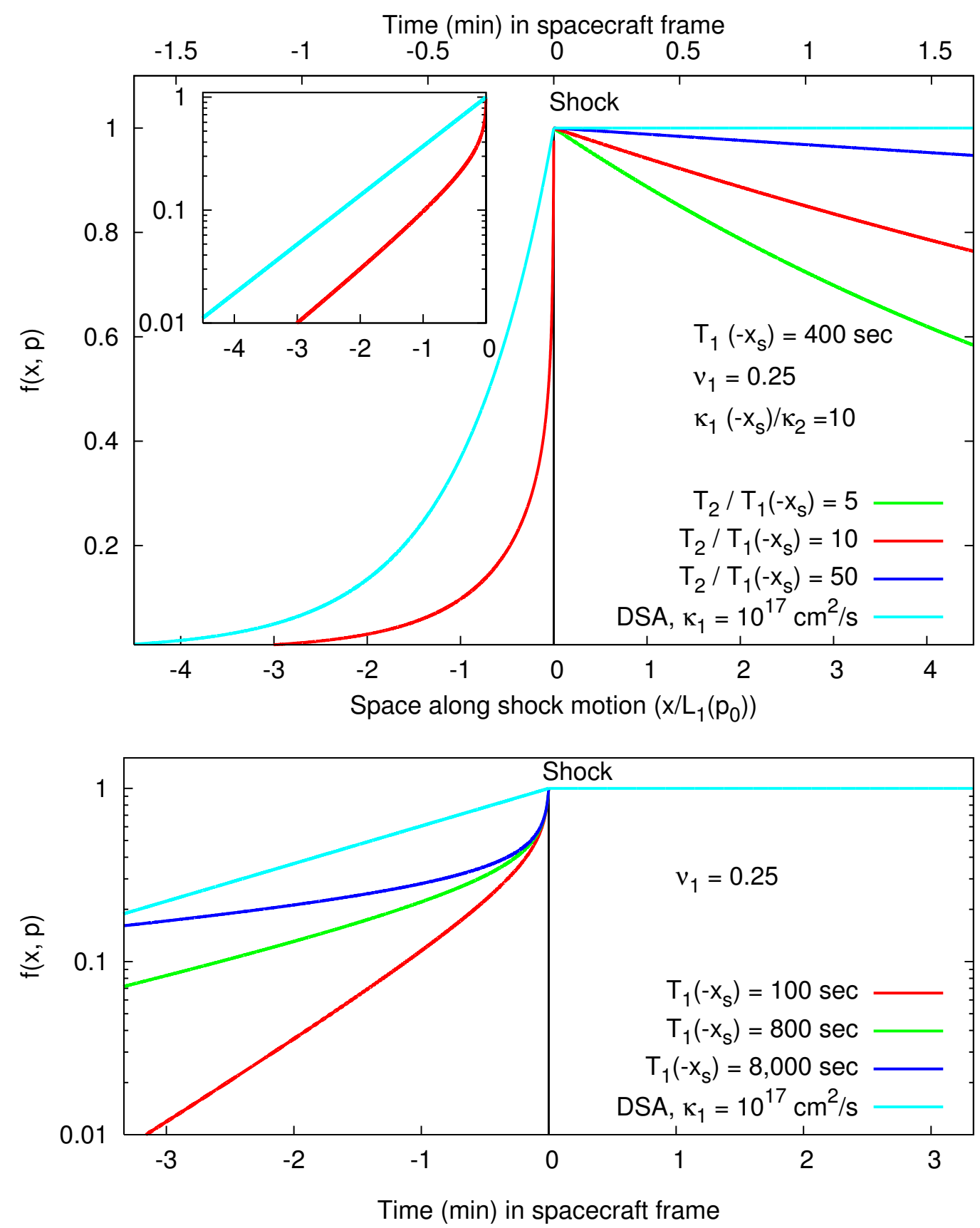

Figure 2. Upper panel: Intensity profiles of energetic particles upstream (Eq. 7) and downstream (Eq. 12) of the shock, normalized to the shock value, for distinct ratios $T_{2}\left(p_{0}\right) / T_{1}\left(-x_{s}, p_{0}\right)$ at a given momentum $p_{0}$ and fixed $\kappa_{1}\left(-x_{s}, p_{0}\right) / \kappa_{2}\left(p_{0}\right)=10$. The DSA profile is shown (in cyan) for comparison with $\kappa_{1}^{D S A}=10^{17} \mathrm{~cm}^{2} / \mathrm{s}$. For $\sim 100 \mathrm{keV}$ protons, we have used $T_{1}\left(-x_{s}, p_{0}\right)=400 \mathrm{sec}, \kappa_{1}\left(-x_{s}, p_{0}\right)=10^{16} \mathrm{~cm}^{2} / \mathrm{s}$ and $U_{1}=500 \mathrm{~km} / \mathrm{s}$ so that $\kappa_{1}^{D S A} / U_{1}=L_{1}\left(p_{0}\right)=2 \times 10^{9} \mathrm{~cm}$. The red curves correspond to the case $\kappa_{1}\left(p_{0}\right) / \kappa_{2}\left(p_{0}\right)=T_{1}\left(p_{0}\right) / T_{2}\left(p_{0}\right)=10$ so that $L_{1}\left(p_{0}\right)=L_{2}\left(p_{0}\right)$. The shock compression is $r=3$. The vertical black line marks the shock location. The lower $x$-axis gives the distance along the shock normal in units of $L_{1}\left(p_{0}\right)$; the upper $x$-axis gives the time in spacecraft frame (assuming a SW speed $=400 \mathrm{~km} / \mathrm{s}$ ). The panel inserted in the top left zooms into the upstream region in logarithmic scale on the $y$-axis. Lower panel: Same as upper panel comparing upstream profiles only (Eq. 7) with DSA profile for distinct $T_{1}\left(-x_{s}, p_{0}\right)$. The $x$-axis gives the time in spacecraft frame.

where we have used $\epsilon / L_{1}(p) \ll 1$ and the $p$-dependence of $\nu_{1}$ through $\kappa_{1}$ is made explicit.

As for the downstream region, the gradient of $f(x, p)$ from Eqs 12:

$$
\left.\frac{\partial f(x, p)}{\partial x}\right|_{+\epsilon}=\left(\frac{U_{2}}{2 \kappa_{2}(p)}-\frac{1}{2} \sqrt{\left(\frac{U_{2}}{\kappa_{2}(p)}\right)^{2}+\frac{4}{L_{2}^{2}(p)}}\right) f(x, p) .
$$




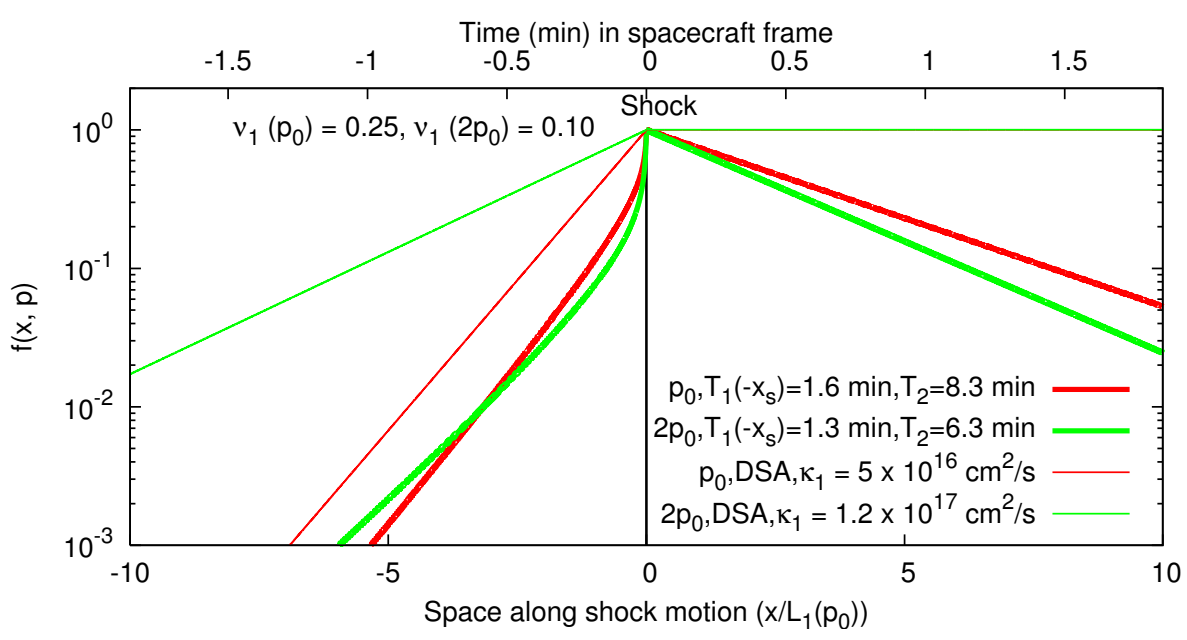

Figure 3. Intensity profiles for distinct particle momenta $p_{0}$ (corresponding to $\sim 100 \mathrm{keV}$, in thick red) and $2 p_{0}$ (in thick green). Here $\kappa_{1}\left(-x_{s}, p_{0}\right)=10^{16} \mathrm{~cm}^{2} / \mathrm{s}, \delta_{1}=1.3, T_{1}\left(-x_{s}, p_{0}\right)=100 \mathrm{sec}, \gamma_{1}=0.4$ and $U_{1}=500 \mathrm{~km} / \mathrm{s}$; we also use $\delta_{1}=\delta_{2}, \gamma_{1}=\gamma_{2}$, $\kappa_{1}\left(-x_{s}, p_{0}\right) / \kappa_{2}\left(p_{0}\right)=10$ and $T_{2}\left(p_{0}\right) / T_{1}\left(-x_{s}, p_{0}\right)=10$. For comparison, the DSA profiles for $p=p_{0}, 2 p_{0}$ are shown as thin curves in red and green, respectively, with scaling $\kappa_{1}^{D S A}\left(p / p_{0}\right)^{\delta_{1}}$ and such that $\kappa_{1}^{D S A}\left(p_{0}\right) / U_{1}=L_{1}\left(p_{0}\right)=10^{9} \mathrm{~cm}$. The shock compression is $r=3$. The vertical black line marks the shock location. The lower $x$-axis gives the distance along the shock normal in units of $L_{1}\left(p_{0}\right)$; the upper $x$-axis gives the time in spacecraft frame (assuming a SW speed $=400 \mathrm{~km} / \mathrm{s}$ ).

For $\nu_{1}<1$, we use the approximation

$$
\frac{\Gamma\left(1-\nu_{1}\right)}{\Gamma\left(1+\nu_{1}\right)}=\frac{\pi}{\nu_{1} \Gamma^{2}\left(\nu_{1}\right) \sin \left(\pi \nu_{1}\right)} \simeq \frac{1}{\nu_{1}^{2} \Gamma^{2}\left(\nu_{1}\right)} .
$$

The source is assumed to be monochromatic and localized at the shock: $S(x, p)=S_{0} \delta(x) \delta\left(p-p_{0}\right)$. The two gradients in Eqs. 15 and 16, replaced into Eq. 14, yield the following equation for $f(p)$, in the limit for $\epsilon \rightarrow 0$ :

$$
\frac{\partial f(p)}{\partial p}-\frac{3 r}{p U_{1}(1-r)}\left[\frac{\kappa_{1}(p)}{\left|\Lambda_{1}\right|} \frac{2}{\nu_{1} \Gamma^{2}\left(\nu_{1}(p)\right)}\left(\frac{\epsilon}{2 L_{1}(p)}\right)^{2 \nu_{1}(p)}-\frac{U_{2}}{2}\left(1-\sqrt{1+\frac{4 \kappa_{2}(p)}{U_{2}^{2} T_{2}(p)}}\right)\right] f(p)+\frac{S_{0}}{U_{1}} \frac{3 r}{1-r} \frac{\delta\left(p-p_{0}\right)}{p}=0 .
$$

By approximating $\Gamma\left(\nu_{1}(p)\right) \sim \frac{1}{\nu_{1}(p)}-C$, where $C$ is the Euler constant (Gradshteyn et al. 2007, Eq. 8.322), and using $\kappa_{2}(p) / U_{2}^{2} T_{2}(p) \ll 1$, we can recast the dominant terms of the solution of Eq. 18 as

$$
f(p) \propto \underbrace{\left(\frac{p}{p_{0}}\right)^{-q} \exp \left[-A_{1}\left(\frac{p}{p_{0}}\right)^{-\delta_{1}}\right]}_{\text {upstream }} \underbrace{\exp \left[-A_{2}\left(\frac{p}{p_{0}}\right)^{\delta_{2}+\gamma_{2}}\right]}_{\text {downstream }}
$$

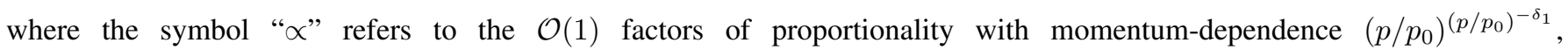
$\exp \left(-\left(p / p_{0}\right)^{-\delta_{1}}\right)$ not included in Eq. 19, the factor $p^{-q}$, with the constant exponent $q=3 r /(r-1)$, is the DSA test-particle power law in the case of no-escape and

$$
\begin{aligned}
& A_{1}=0.4 \frac{q}{\delta_{1}} \frac{U_{1}}{400 \mathrm{~km} / \mathrm{s}} \frac{\left|\Lambda_{1}\right|}{10^{11} \mathrm{~cm}}\left(\frac{\bar{\kappa}_{1}}{10^{19} \mathrm{~cm}^{2} / \mathrm{s}}\right)^{-1}\left|-5.3+\log \frac{\epsilon / 10^{7} \mathrm{~cm}}{L_{1}\left(p_{0}\right) / 10^{9} \mathrm{~cm}}\right|, \\
& A_{2}=6.25 \times 10^{-5} \frac{q}{r\left(\delta_{2}+\gamma_{2}\right)} \frac{\bar{\kappa}_{2}}{10^{15} \mathrm{~cm}^{2} / \mathrm{s}}\left(\frac{U_{2}}{400 \mathrm{~km} / \mathrm{s}}\right)^{-2}\left(\frac{\bar{T}_{2}}{10^{4} \mathrm{sec}}\right)^{-1}
\end{aligned}
$$

where we have used $\bar{T}_{2}=\bar{T}_{1}\left(0, p_{0}\right)$. We note that the expression for $f(p)$ in Eq. 19 does not depend on $\gamma_{1}$ : the momentum spectrum does not depend on the assumption on the upstream power law index of $T$ in momentum provided that $\delta_{1}>\gamma_{1}$ and $\delta_{1}+\gamma_{1}>1$. The width of the peak of the spectrum at low $p$ is controlled by the constant $A_{1}$, namely by $U_{1} \Lambda_{1}\left(\log L_{1}\right) / \bar{\kappa}_{1}$, whereas the drop at large $p$ is controlled by the constant $A_{2}$, namely by $\bar{\kappa}_{2} / U_{2}^{2} \bar{T}_{2}$. Table 1 lists some values of $A_{1}, A_{2}$ for illustrative purpose. 
Figure 4 compares the spectrum in Eq. 19 for distinct $T_{1}\left(-x_{s}, p_{0}\right)$ with DSA test-particle power-law and with the log-parabola spectrum $\left(\propto\left(p / p_{0}\right)^{-\alpha-\beta \log \left(p / p_{0}\right)}\right)$ that fits in all panels the green curve, corresponding to the case $T_{1}\left(-x_{s}, p_{0}\right)=3,600$ sec, where the curvatures $\beta$ span the range of the best-fit values for the spectra of the 16 GLE events during solar cycle 24 (Zhou et al. 2018). Figure 4 also depicts the phase-space distribution function $f_{B}(p)$, corresponding to the Band function customarily used to fit the measured differential intensity, i.e., $d J / d E=p^{2} f_{B}(p)$ for non-relativistic energy, that can be recast as

$$
f_{B}(p) \propto \begin{cases}\left(p / p_{0}\right)^{2\left(\zeta_{1}-1\right)} \exp \left(-\left(p / p_{b}\right)^{2}\right) & \text { if } p<\sqrt{\zeta_{1}-\zeta_{2}} p_{b} \\ \left(p / p_{0}\right)^{2\left(\zeta_{2}-1\right)}\left[\left(\zeta_{1}-\zeta_{2}\right)\left(p_{b} / p_{0}\right)^{2}\right]^{\zeta_{1}-\zeta_{2}} \exp \left(\zeta_{2}-\zeta_{1}\right) & \text { if } p>\sqrt{\zeta_{1}-\zeta_{2}} p_{b}\end{cases}
$$

where $\zeta_{1}$ and $\zeta_{2}$ are power law indexes of $d J / d E$ as a function of the energy rather than $p$. In Fig. $4 f_{B}(p)$ is chosen to fit the asymptotic slopes of the log-parabola at low- and large-momentum .

The parameters of the log-parabola $\alpha, \beta$ are related to the probability of particle escape from the acceleration region; in particular, the curvature $\beta$ is related to $R=E / E_{0}$, i.e., the energy gain in the single shock-crossing with initial (final) energy $E_{0}$ $(E): \beta=q^{\prime} / 2 \log R$, where $q^{\prime}$ is a parameter of the probability of escape $\mathcal{P}(E)=g / E^{q^{\prime}}$ where $g$ is a constant (Massaro et al. 2004; Fraschetti \& Pohl 2017a).

The determination of the microscopic parameters $\alpha, \beta$ (and $\zeta_{1}, \zeta_{2}$ ) in terms of the new constants $A_{1}, A_{2}$, that depend on macroscopic transport and shock parameters, requires the determination of constant coefficients in a transcendental equation. The graphic solution in Fig. 4 suggests the best-fit correspondence between $\alpha, \beta$ (or $\zeta_{1}, \zeta_{2}$ ) and $A_{1}, A_{2}, \delta_{i}$ for distinct $\gamma_{i}$. Figure 4 , b) shows that the asymptotic slopes of the Band curve emerge, over almost 3 decades in momentum (i.e., $>5$ decades in kinetic energy), for large values of $T_{1}\left(-x_{s}, p\right) \simeq 1$ hour, large $U_{1} \simeq 250 \mathrm{~km} / \mathrm{s}$, small index $\gamma_{2}=0.4$ and $\kappa_{1}\left(-x_{s}, p_{0}\right) / \kappa_{2}\left(p_{0}\right)=10$. In all panels the Band function overestimates the spectrum from both other models at $p \simeq p_{b}$, allowing to discriminate between models $^{2}$ (Zhou et al. 2018). Likewise, in the escape-free DSA, the spectral power-law index can be expressed both as a function of the macroscopic density compression and, alternatively, as a function of microscopic quantities, i.e., of the probability of remaining in the acceleration region $(P)$ and of $R: \log P / \log R$.

\begin{tabular}{|l|c|c|c|c|c|}
\hline \multicolumn{2}{|c|}{} & \multicolumn{2}{c|}{$\gamma_{1}=\gamma_{2}=0.4$} & \multicolumn{2}{c|}{$\gamma_{1}=\gamma_{2}=0.7$} \\
\hline$U_{1}(\mathrm{~km} / \mathrm{s})$ & $T_{1}\left(-x_{s}, p_{0}\right)(\mathrm{s})$ & $A_{1}$ & $A_{2}$ & $A_{1}$ & $A_{2}$ \\
\hline \multirow{3}{*}{100} & 100 & 2.38 & 0.0964 & 2.38 & 0.0794 \\
& 300 & 2.63 & 0.0321 & 2.63 & 0.0265 \\
& 3,600 & 3.19 & 0.00268 & 3.19 & 0.00221 \\
\hline \multirow{3}{*}{250} & 100 & 5.96 & 0.0154 & 5.96 & 0.0127 \\
& 300 & 6.58 & 0.00514 & 6.58 & 0.00424 \\
& 3,600 & 7.98 & 0.000429 & 7.98 & 0.000353 \\
\hline
\end{tabular}

Table 1. Spectral parameters $A_{1}, A_{2}$ for the cases depicted in Fig. 4 are summarized in terms of $U_{1}, T_{1}$ and $\gamma_{i}$.

\section{DISCUSSION}

The solution in Eq. 19 provides a simple no-power-law momentum spectrum of energetic particles, remarkably different from the power-law + exponential cutoff and can be mapped into a log-parabola. The log-parabola parameters $\alpha$ and $\beta$ and the Band function parameters $\zeta_{1}, \zeta_{2}$ and $p_{b}$ are related via $A_{1}, A_{2}$ (see Eq.s 21) to the DSA test-particle power-law index $q$, the upstream shock speed $U_{1}$, the far-upstream spatial diffusion coefficient $\bar{\kappa}_{1}$, the length-scale $\Lambda_{1}$ where the self-generated turbulence is drowned into the pre-existing far upstream turbulence and logarithmically by the escape length $L_{1}\left(p_{0}\right)$. In contrast, in the powerlaw + exponential cutoff solution the roll-over length-scale depends on the diffusion properties close to the shock. Due to the weak (logarithmic) dependence of $A_{1}$ on $L_{1}\left(p_{0}\right)$, the value of $L_{1}\left(p_{0}\right)$ is likely to be constrained via the in-situ measured intensity profiles rather than the spectra. In the Appendix the power-law+exponential cutoff spectrum (Ellison \& Ramaty 1985) is shown to be retrieved in the limit of acceleration faster than escape $\left(\kappa_{1}(p) / U_{1}^{2} T_{1}(p) \ll 1\right)$ by solving Eq. 1 with $\kappa$ and $T$ spatially independent throughout, provided a discontinuous jump at the shock.

\footnotetext{
${ }^{2}$ The dependence of the energy break on the ions charge-to-mass ratio (Mewaldt 2006; Desai et al. 2016) can be expressed as dependence of $\beta$ on the ions chargeto-mass ratio via the anti-correlation $\beta \simeq E_{b}^{-0.36}$, determined only for the 16 GLE of solar cycle 24 (Zhou et al. 2018), where $E_{b}$ is the Band function energy break.
} 


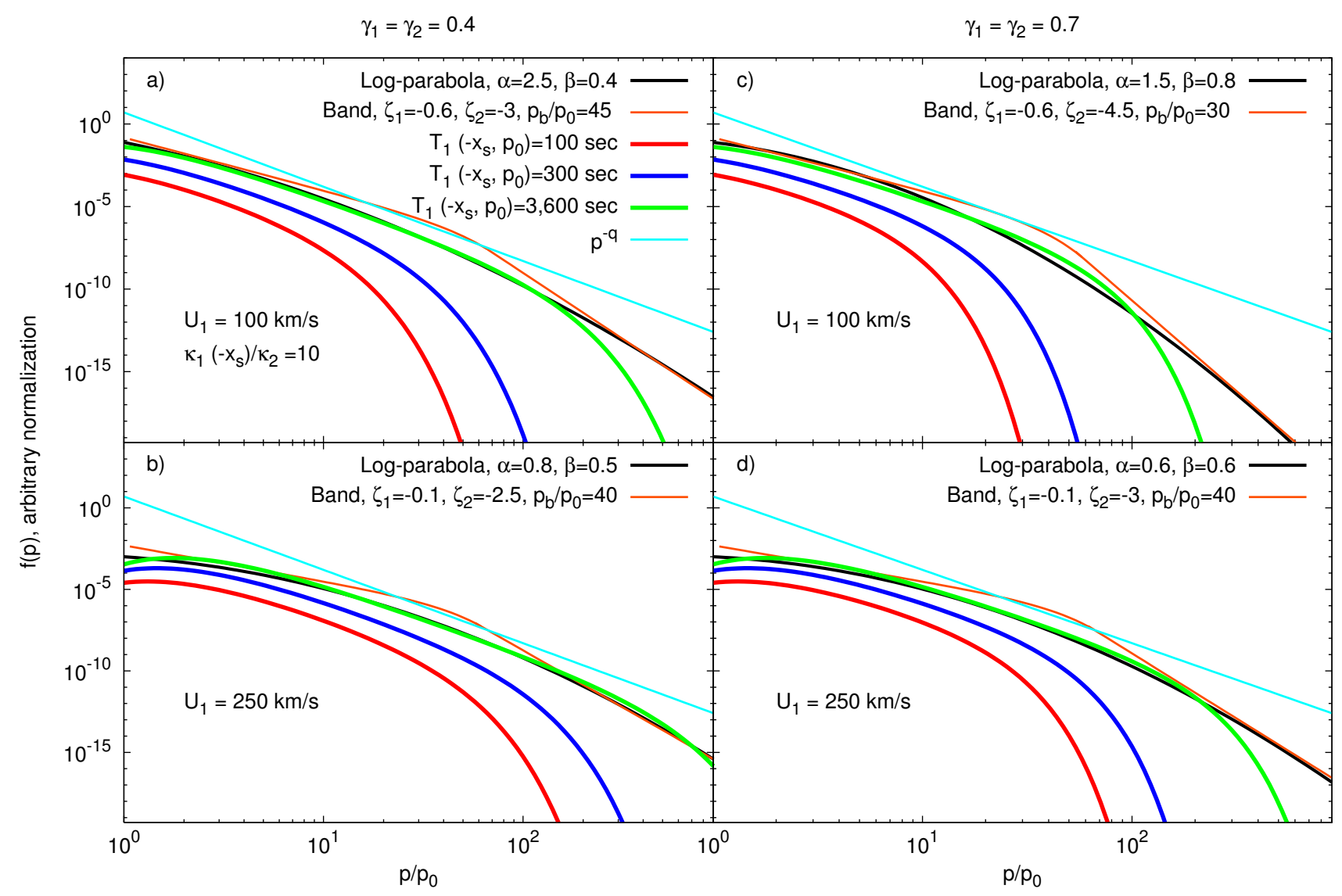

Figure 4. a): Momentum spectrum in Eq. 19 for distinct values of $T_{1}\left(-x_{s}, p_{0}\right)=100 \mathrm{sec}$ (in red), $300 \mathrm{sec}$ (in blue) and 3, $600 \mathrm{sec}$ (in green), compared with the test-particle DSA solution (in cyan), with a log-parabola (in black) reproducing the spectrum in the case $T_{1}\left(-x_{s}, p_{0}\right)=$ $3,600 \mathrm{sec}$ (in this panel up to $p / p_{0} \simeq 150$ that corresponds to $\simeq 4$ decades in particle energy), and with a Band function (in orange) for illustrative purpose with asymptotic slopes fitting the log-parabola. Here $\bar{\kappa}_{1}=10^{19} \mathrm{~cm}^{2} / \mathrm{s},\left|\Lambda_{1}\right|=10^{11} \mathrm{~cm}, r=3, U_{1}=100 \mathrm{~km} / \mathrm{s}$, $\delta_{1}=\delta_{2}=1, \gamma_{1}=\gamma_{2}=0.4, \kappa_{1}\left(-x_{s}, p_{0}\right) / \kappa_{2}\left(p_{0}\right)=10$ and in all cases $T_{2}\left(p_{0}\right) / T_{1}\left(-x_{s}, p_{0}\right)=10$ so that $\kappa_{2}(p) / U_{2}^{2} T_{2}(p) \ll 1$. The blue and red curves are multiplied by arbitrary factors, respectively by 0.1 and 0.01 , for readability purposes. $b$ ): Same as $a$ ) with $U_{1}=250 \mathrm{~km} / \mathrm{s}$. $c$ ): Same as $a$ ) with $\gamma_{1}=\gamma_{2}=0.7 . d$ ): Same as $c$ ) with $U_{1}=250 \mathrm{~km} / \mathrm{s}$.

The value of $\gamma$ is constrained by the two requirements that for higher $p$ (see Sect. 4.3) the intensity profiles extend further upstream $(\gamma<\delta$, or $d L / d p>0)$ and that the ratio of acceleration to escape time scale grows with momentum $\left(\delta_{1}+\gamma_{1}>1\right.$, realized for a $3 \mathrm{D}$ isotropic turbulence or in the Bohm limit). Further constraints on $T$ and on its spatial dependence both at the shock and far from it can be identified via direct numerical simulations that are beyond the scope of this work. In addition, $\gamma$ is likely to be affected by the nature of the magnetic turbulence, as much as $\delta$; a specific turbulence power spectrum is not implemented herein for the sake of simplicity. The fact that $\delta \neq \gamma$ might seem at odds with the assumption that the upstream spatial dependences of $\kappa$ and $T$ are reciprocal: if both $\kappa$ and $T$ are governed by the same turbulence, $\delta$ and $\gamma$ should be nearly equal. An argument supporting the reciprocal spatial dependence, beside the higher density of scattering centers close to the shock, is that the only quantity with dimension of length that combines $\kappa$ and $T$, that is $L(p)$, is expected to be weakly dependent on space (in Eqs. 2 and 3 it is exactly independent of $x$ ), as much as the diffusion length (for a uniform $U_{1}$ ); on the other hand, $L_{1}(p) \propto\left(p / p_{0}\right)^{\left(\delta_{1}-\gamma_{1}\right) / 2}$ is expected to depend on $p$ to account for the observed larger extent of the diffusive streaming ahead of the shock at larger $p$; thus, $\delta_{1} \neq \gamma_{1}$. Alternatively, a momentum-dependence of the escape time can be derived from confinement arguments, by assuming a Sedov-type time dependence for the maximum momentum of the energetic particles before leaving the shock, with no return to it both from upstream and from downstream (Ptuskin \& Zirakashvili 2005; Celli et al. 2019). 
In the context of the escape of cosmic rays from the galaxy, Lerche \& Schlickeiser (1985) concluded that an agreement with the observed trend of the secondaries-to-primaries cosmic rays flux ratio at energies $>10 \mathrm{GeV} /$ nucleon can be achieved, for primaries accelerated both by shocks and by momentum diffusion off Alfvénic turbulence, only if both $\delta$ and $\gamma$ (respectively $\eta$ and $b$ therein) are non-zero and positive. The case $\delta \neq \gamma$ for the primary cosmic rays was not ruled out by observations. Modelling of non-thermal electron emission in solar flares also makes use of a distinct momentum-scaling of $\kappa$ and $T$ (Petrosian 2016).

The model introduced herein can be regarded as an extension of the leaky-box model. Typically an escape time is introduced to solve separately the transport equation in the momentum space and enters as eigenvalue in a series expansion of the steady-state transport equation with a FEB (Schlickeiser 2002, chap. 14). In the model presented here, the space and momentum dependence of $\kappa$ prevents the separate solution of the transport equations for position and momentum. Nevertheless, it is shown here that a spatial solution can be determined analytically, if the upstream is populated by a self-generated turbulence that at a distance $\left|\Lambda_{1}\right|$ from the shock is taken over by pre-existing turbulence rather than vanishing at a FEB.

Discontinuous changes in the $f(x, p)$ such as charge exchange (or leptons pair annihilation) are not included in the term $f / T$ in Eq. 1. For protons, the charge exchange with thermal upstream hydrogen atoms, using a SW density $\sim 1 \mathrm{~cm}^{-3}$ and a relative speed $\lesssim 1,000 \mathrm{~km} / \mathrm{s}$ that is much larger than the shock speed in the frame of the upstream interplanetary medium, has ${ }^{3}$ time scale $\sim 10^{6} \mathrm{sec}$. For charge exchange protons/heavier ions (such as C,O), such a time scale is of order $10^{7}-10^{8} \mathrm{sec}$. Thus, the charge exchange time scales are much longer than any time scale of interest for interplanetary shocks; however, they are relevant for longer-lived (e.g., interstellar) shocks. For ions fragmentation due to ion-ion collisions or spallation, the time scale is much longer than $\sim$ years at the SW density and in the particle energy range considered here $(0.1-1 \mathrm{GeV}$; Silberberg \& Tsao 1990). These processes are therefore also not included in $T$ for interplanetary shocks although they need to be included for interstellar shocks.

We propose that the particles escaped from the shock and diffusing into the SW are described by a diffusion equation that does contain the loss term $f / T$ hereby introduced. However, the scale of the acceleration region that particles escape from might exceed by several orders of magnitude the scale of the shock region, close to the scale of the heliosphere, thereby making the $f / T$ term negligible: the escape time is much larger than any other relevant time scale for processes occurring during the propagation of the particles, e.g., not only charge exchange but also adiabatic energy losses, reacceleration by another shock, trapping into planetary magnetosphere, etc. In addition, the escape term might be relevant to identify the source of the shock-accelerated particles measured as SEP (at 1 AU but also at Parker Solar Probe or Solar Orbiter much closer to the Sun ) arriving hours before the shock that produced them. Equation 1 can be helpful to model such an "SEP escape".

Finally, the approach presented herein does not aim at describing the formation of the spectral energy tail from an initial single Maxwellian distribution; this process is egregiously being tackled by Particle-In-Cell (PIC) simulations despite over limited spatial and temporal domains (see e.g., Pohl et al. 2020, and references therein). On the other hand the gyroradius and energy scales of the majority of the escaping particles are beyond reach of the current PIC capabilities. Test-particles numerical simulations are a suitable investigation tool to measure numerically the absolute value and the scaling in position and momentum of the $T$ as herein introduced, but they are beyond the scope of this work.

\section{CONCLUSION}

We have built a steady-state 1D transport model for energetic particles at shocks allowing for a finite acceleration-to-escape time scale ratio upstream at any distance from the shock and at any particle energy, with no imposed energy-independent escape boundary. By using a hybrid spatial dependence of the diffusion coefficient, i.e., linearly increasing with the distance from the shock upstream to account for the self-generated turbulence due to streaming ions (Bell 1978) and uniform downstream, we find intensity profiles and momentum spectra of energetic particles remarkably different from the DSA solution. As for the intensity profiles, the steep drop upstream of the shock suggests an interpretation of the supra-thermal ions events in the Earth bow shock multi-spacecraft observations (Kis et al. 2004). Although a large SW local velocity might explain the short roll-over distance in front of the shock, the model for the escape presented here offers a possible alternative that deserves to be explored and compared with data in detail. Far upstream, at a distance comparable with the diffusion scale, the profile recovers the DSA exponential roll-over, although with a reduced amplitude. The downstream profile is uniform (as in DSA) for downstream escape time larger than the advection time and drops from the shock more steeply than DSA as the escape time becomes comparable with the local advection time.

${ }^{3}$ We use the International Atomic Energy Agency database http: //www-amdis.iaea.org/ALADDIN/. 
We have provided a derivation of the 1D momentum spectrum that has the form of the product of a power law and two exponentials and can be mapped into a log-parabola. This model offers a derivation of the log-parabola spectrum complementary to the probability-based derivation used, e.g., in Massaro et al. (2004); Fraschetti \& Pohl (2017a). In summary, the power-law scaling of the momentum spectrum, that results from a process of particle energization increasing at higher energy (dating back to the original idea in Fermi 1949) is extended herein to a more general scaling of a power law modulated by two exponentials; such a new form of the spectrum matches within a certain parameter range the log-parabola spectrum, that results from allowing particle escape at any energy/distance from the shock, not only at the highest energy nor only at a given spatial boundary. We emphasize that the log-parabola does not replace the power law, applicable in narrower energy ranges, but rather extends it to a broader energy range.

Multi-dimensional effects (shock rippling, large-scale shock curvature, finite extent of the shock surface not considered here) also contribute to reshaping the high-energy part of the spectrum and were considered in Drury (2011); Malkov \& Aharonian (2019). The effect of shock corrugation at the highest energy ion scale on the escape can be analytically included in this model (Fraschetti 2013, 2014) and comparison with multi-spacecraft measurements can be used to constrain the momentum dependence of $T$.

The prospect of measuring with Parker Solar Probe and Solar Orbiter the escape of $0.1-1$ GeV particles åccelerated at very high speed shocks, more likely to be crossed close to the Sun than at 1 AU, opens new opportunities to test the model herein presented. So far only a handful of remarkably weak shock events have crossed either spacecraft, but the ongoing increase of the solar activity toward the next maximum is expected to lead to observations in an unchartered physical domain.

\section{ACKNOWLEDGMENTS}

The constructive comments of the referee are gratefully acknowledged. This work was supported, in part, by NSF under grant 1850774 , by NASA under Grant 80NSSC18K1213 and by Chandra Theory grant GO8-19015X. FF thanks Drs. J. Giacalone and J. Kota for discussions and suggestions and Drs. A. M. Bykov, L. Drury and M. W. Pohl for comments in an email exchange.

\section{APPENDIX}

\section{A. SPATIAL PROFILE: CASE OF SPATIALLY UNIFORM $\kappa$ AND $T$}

The shock is assumed not to significantly perturb the medium it is propagating into so that the upstream parameters of the turbulence can be assumed to hold pre-existing values and are unchanged with the distance from the shock. Thus, we can use a spatial diffusion coefficient $\kappa$ uniform in space both upstream and downstream and discontinuous at the shock only:

$$
\kappa(x, p)=\left\{\begin{array}{l}
\kappa_{1}(p) \text { if } x<0, \text { upstream } \\
\kappa_{2}(p) \text { if } x>0, \text { downstream } .
\end{array}\right.
$$

Likewise, the escape time $T$ is assumed to be discontinuous at the shock and uniform elsewhere:

$$
T(x, p)=\left\{\begin{array}{l}
T_{1}(p) \text { if } x<0, \text { upstream } \\
T_{2}(p) \text { if } x>0, \text { downstream } .
\end{array}\right.
$$

The transport equation 1 with the assumptions in Eqs. A1, A2 is solved in coordinates-space as follows.

\section{A.1. Upstream spatial profile}

We seek the general solution upstream $\left(U=U_{1}\right.$ so that $d U / d x=0$ and far from the source $\left.S(x, p)=0\right)$ of the equation

$$
U_{1} \frac{\partial f(x, p)}{\partial x}=\kappa_{1}(p)\left[\frac{\partial^{2}}{\partial x^{2}} f(x, p)\right]-\frac{f(x, p)}{T(p)} .
$$

We impose two boundary conditions to determine the two integration constants. The first one gives the value of $f$ at the shock $f(0, p)=f_{0}(p)$. The second integration constant is chosen to prevent exponential divergence. The upstream solution is

$$
f(x, p)=f_{0}(p) \exp \left[\left(\frac{U_{1}}{2 \kappa_{1}(p)}+\frac{1}{2} \sqrt{\left(\frac{U_{1}}{\kappa_{1}(p)}\right)^{2}+\frac{4}{L_{1}^{2}(p)}}\right) x\right]
$$


that tends to $\exp \left(U_{1} x / \kappa_{1}(p)\right)$ as $T_{1}(p) \rightarrow \infty$, i.e., with identical spatial dependence as the upstream profile in case of no particleescape (DSA) and in case of space-independent $\kappa$ and $U$. Equation A4 shows that if the upstream $\kappa$ is uniform, the effective diffusion length $\left(\frac{U_{1}}{2 \kappa_{1}(p)}+\frac{1}{2} \sqrt{\left(\frac{U_{1}}{\kappa_{1}(p)}\right)^{2}+\frac{4}{L_{1}^{2}(p)}}\right)^{-1}$ is smaller than $\kappa_{1} / U_{1}$ for any $T_{1}$. Unlike the case of no-escape, where the asymptotic limit at $x \rightarrow-\infty$ is a non-vanishing constant depending only on momentum (that also solves Eq. A3 in the limit $T_{1}(p) \rightarrow \infty$ ), the solution in Eq. A4 tends exponentially to zero far upstream, due to the escape. An exponential dilution of particles is also found in the case of spatially dependent $\kappa$ (see Sect. 4). Likewise, particles do not disappear but spread out to an infinite distance with an exponentially small amplitude.

\section{A.2. Downstream spatial profile}

In the downstream region ( $U=U_{2}$ so that $d U / d x=0$ and far from the source $S(x, p)=0$ ) we impose the continuity at the shock with the upstream solution: $f(0, p)=f_{0}(p)$. The second integration constant is again chosen to prevent exponential divergence. The solution is then

$$
f(x, p)=f_{0}(p) \exp \left[\left(\frac{U_{2}}{2 \kappa_{2}(p)}-\frac{1}{2} \sqrt{\left(\frac{U_{2}}{\kappa_{2}(p)}\right)^{2}+\frac{4}{L_{2}^{2}(p)}}\right) x\right]
$$

with limit $f_{0}(p)$ in the case of no-escape $\left(T_{2}(p) \rightarrow \infty\right)$, as expected. Both upstream and downstream profiles depend on the ratio of $\kappa(p) / U$ (upstream coinciding with the diffusion length) to $L(p)=\sqrt{\kappa(p) T(p)}$ : if $T(p)$ is very large, one recovers the DSA solution.

Examples of spatial profiles are illustrated in Fig. 5 for $T_{1}=100 \mathrm{sec}$ and distinct $T_{2}$ (upper panel), for $T_{1}=5 \mathrm{sec}$ and distinct $T_{2}$ (lower panel), both at a given particle momentum $p_{0}$ corresponding to $\sim 100 \mathrm{keV}$ protons; here $\kappa_{1}\left(p_{0}\right)=10^{16} \mathrm{~cm}^{2} / \mathrm{s}(\mathrm{Kis}$ et al. 2018). The DSA profile is drawn for comparison (in cyan). The upstream profile is close to the DSA exponential roll-over for large $T_{1}=100 \mathrm{sec} \gg x / U_{1} \sim 10 \mathrm{sec}$ (Fig. 5, upper panel): in this case the diffusion scale is $\kappa_{1}\left(p_{0}\right) / U_{1}=2.5 \times 10^{8} \mathrm{~cm}$ and $L_{1}=10^{9} \mathrm{~cm}$. For $T_{1}=5 \mathrm{sec}<x / U_{1}$ (Fig. 5, lower panel) the profile steepens with respect to the DSA prediction (here $\left.L_{1}=\sqrt{\kappa_{1} T_{1}}<\kappa_{1} / U_{1}\right)$. In both panels, large downstream escape times $\left(T_{2} \gtrsim 1,000 \mathrm{sec}\right.$, upper panel, blue and black curves, and lower panel, black curve) with respect to the advection time $x / U_{2} \simeq 30 \mathrm{sec}$ lead to a profile comparable with the uniform prediction of DSA, as the escape becomes irrelevant and the profile is advection-dominated. For $T_{2} \sim 25 \sec <x / U_{2}$ (lower panel, green curve) the profile drops behind the shock due to particles moving downstream away from the shock faster than the advection and not efficiently back-scattering to return to the shock.

\section{B. MOMENTUM SPECTRUM: CASE OF SPATIALLY UNIFORM $\kappa$ AND $T$}

From the continuity of the spatial profiles across the shock (see Sect. A), the momentum spectrum at the shock $f_{0}(p)=f(p)$ can be found by following the usual textbook derivation in the case of no-escape. The number of particles flowing along the $\mathrm{x}$ direction has to be continuous across the shock: $\int_{-\epsilon}^{+\epsilon} d x U \partial f / \partial x=0$, for an infinitesimally small $\epsilon$. From Eq. 1, the continuity simply reads:

$$
\left.\left[\kappa(p) \frac{\partial f(x, p)}{\partial x}+\frac{1}{3} U p \frac{\partial f(x, p)}{\partial p}\right]\right|_{-\epsilon} ^{+\epsilon}+\int_{-\epsilon}^{+\epsilon} d x\left[S(x, p)-\frac{f(x, p)}{T(p)}\right]=0 .
$$

By calculating the upstream and downstream gradients of $f$ from Eqs. A4 and A5, using a mono-chromatic source at the shock, i.e., $S(x, p)=S_{0} \delta(x) \delta\left(p-p_{0}\right)$, and replacing the two gradients into Eq. B6 yields the following equation for $f(p)$, in the limit of $\epsilon \rightarrow 0$ :

$$
\frac{\partial f(p)}{\partial p}-\frac{3 r}{2 p(1-r)}\left[1+\sqrt{1+\frac{4 \kappa_{1}(p)}{U_{1}^{2} T_{1}(p)}}-\frac{1}{r}\left(1+\sqrt{1+\frac{4 \kappa_{2}(p)}{U_{2}^{2} T_{2}(p)}}\right)\right] f(p)+\frac{S_{0}}{U_{1}} \frac{3 r}{1-r} \frac{\delta\left(p-p_{0}\right)}{p}=0
$$

where we have used that $\int_{-\epsilon}^{+\epsilon} d x f(x, p) / T(p)=0$. The general solution has a cumbersome logarithmic form. For momentum small enough that the limit $\kappa_{i}(p) / U_{i}^{2} T_{i}(p) \ll 1$ is satisfied both upstream and downstream $(i=1,2)$, i.e., acceleration time scale shorter than escape time scale, the solution has an interesting form. By assuming the momentum dependence for $\kappa_{i}(p)$ and $T_{i}(p)$ in Eqs. 13, we recast the solution as

$$
f(p) \propto\left(\frac{p}{p_{0}}\right)^{-q} \exp \left[-\frac{q}{\delta_{1}+\gamma_{1}} \frac{\bar{\kappa}_{1}}{U_{1}^{2} \bar{T}_{1}}\left(\frac{p}{p_{0}}\right)^{\delta_{1}+\gamma_{1}}\right] .
$$



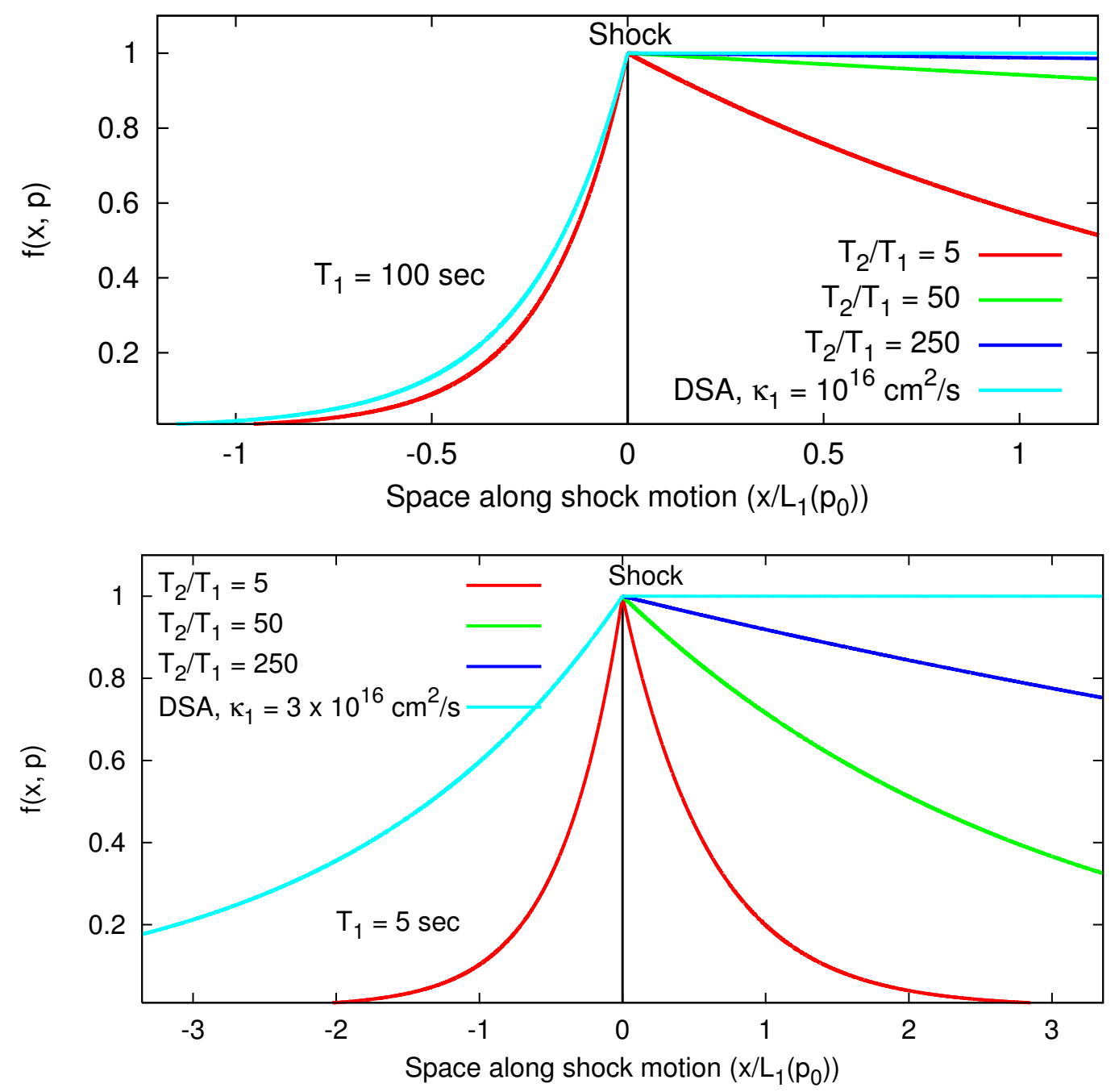

Figure 5. Upper panel: Spatial profiles in the case of uniform $\kappa$ and $T$ for a given momentum $p_{0}$ of the accelerated protons (corresponding to $\sim 100 \mathrm{keV}$ ) for fixed $T_{1}\left(p_{0}\right)=100 \mathrm{sec}$ and distinct $T_{2} / T_{1}\left(p_{0}\right)$; here we choose $\kappa_{1}\left(p_{0}\right) / \kappa_{2}\left(p_{0}\right)=5, \kappa_{1}\left(p_{0}\right)=10^{16} \mathrm{~cm}^{2} / \mathrm{s}$ and $U_{1}=400$ $\mathrm{km} / \mathrm{s}$ so that the diffusion scale is $\kappa_{1}\left(p_{0}\right) / U_{1}=2.5 \times 10^{8} \mathrm{~cm}$ and $L_{1}=10^{9} \mathrm{~cm}$ (see Eq. A4). The red curves correspond to the case $\kappa_{1}\left(p_{0}\right) / \kappa_{2}\left(p_{0}\right)=T_{1}\left(p_{0}\right) / T_{2}\left(p_{0}\right)=5$ so that $L_{1}\left(p_{0}\right)=L_{2}\left(p_{0}\right)$. The shock compression is $r=3$. The DSA profile at $p=p_{0}$ is shown in cyan for comparison. Lower panel: Same as upper panel with $T_{1}\left(p_{0}\right)=5 \mathrm{sec}, \kappa_{1}\left(p_{0}\right)=3 . \times 10^{16} \mathrm{~cm}^{2} / \mathrm{s}$ and $\kappa_{1}\left(p_{0}\right) / U_{1}=7.5 \times 10^{8} \mathrm{~cm}$ $>L_{1}=3.9 \times 10^{8} \mathrm{~cm}$.

where we have used $\kappa_{1}(p) / T_{1}(p) \gg \kappa_{2}(p) / T_{2}(p)$; the exponential roll-over is governed by power-laws exponents of $\kappa$ and $T$ combined: $\delta_{1}+\gamma_{1}$. In Eq. B8 the power-law + exponential roll-over solution (Ellison \& Ramaty 1985) is retrieved, with steeper drop for larger $\bar{\kappa}_{1}$, as expected. Figure 6 (left and middle panels) depicts the spectrum in Eq. B8 for two cases: 1) for fixed $\kappa_{1}\left(p_{0}\right), \gamma_{1}$ and distinct $\left.\delta_{1} ; 2\right)$ fixed $\delta_{1}$ and distinct $\kappa_{1}\left(p_{0}\right)$.

In the opposite limit, i.e., $\kappa_{i}(p) / U_{i}^{2} T_{i}(p) \gg 1$, the resulting spectrum is (see Fig. 6, right panel):

$$
f(p) \propto\left(p / p_{0}\right)^{-3 / 2} \exp \left[-\frac{2 q}{\delta_{1}+\gamma_{1}} \sqrt{\frac{\bar{\kappa}_{1}}{U_{1}^{2} \bar{T}_{1}}}\left(p / p_{0}\right)^{\frac{\delta_{1}+\gamma_{1}}{2}}\right] .
$$

In this case, the acceleration is so inefficient that the spectrum is suppressed exponentially more vigorously than the solution in Eq. B8 (this condition reads $\delta_{1}+\gamma_{1}<2$ ).

\section{REFERENCES}

Acero, F., Ackermann, M., Ajello, M., et al. 2015, ApJS, 218, 23, doi: 10.1088/0067-0049/218/2/23
Armstrong, J. W., Rickett, B. J., \& Spangler, S. R. 1995, ApJ, 443, 209, doi: $10.1086 / 175515$ 


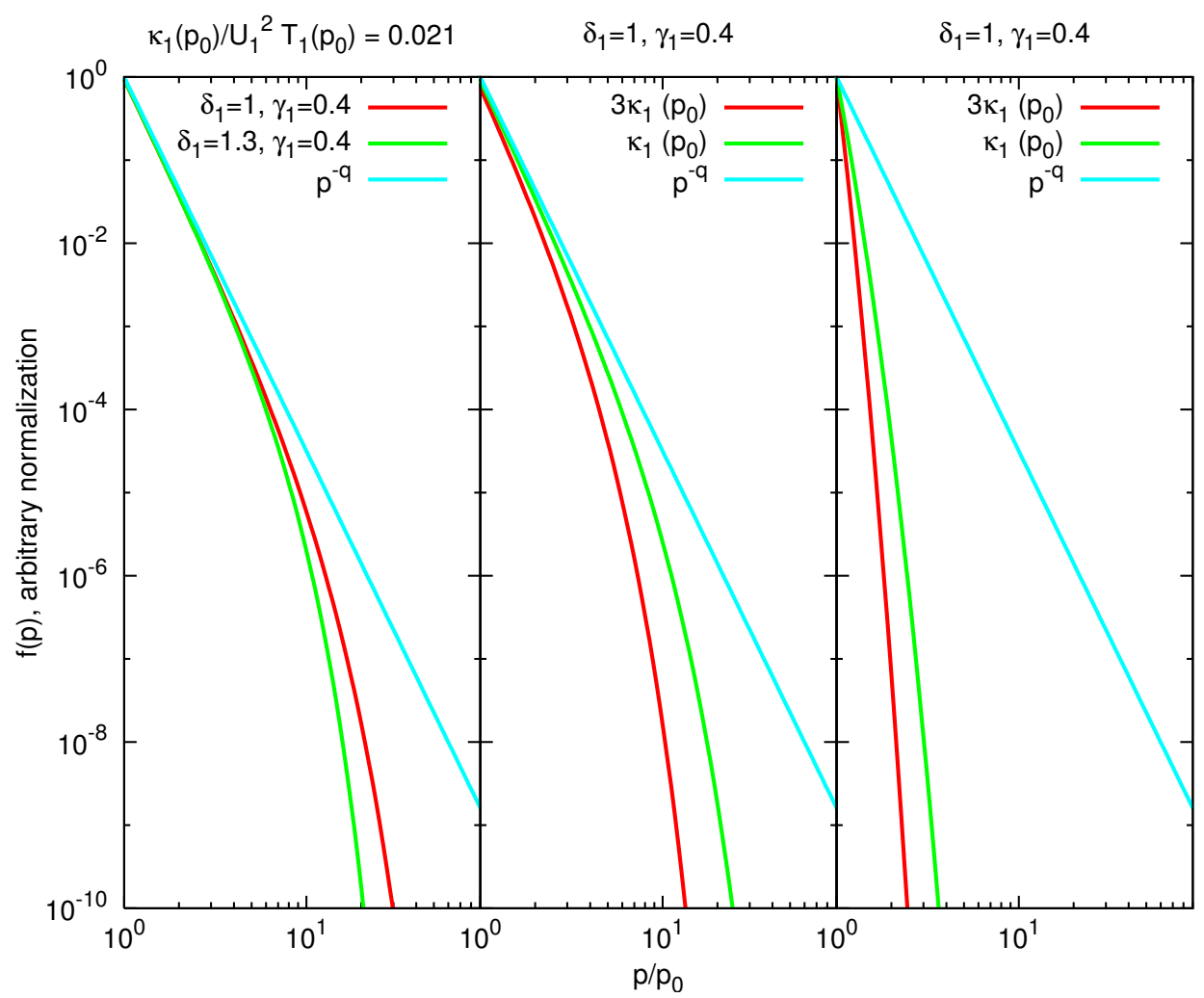

Figure 6. Left panel: Spectrum in the case of uniform $\kappa$ and $T$ for distinct values of $\delta_{1}$ and $\kappa_{1}\left(p_{0}\right) / U_{1}^{2} T_{1}\left(p_{0}\right) \ll 1$, compared with the testparticle DSA solution (in cyan). Here $\kappa_{1}\left(p_{0}\right)=10^{16} \mathrm{~cm}^{2} / \mathrm{s}, T_{1}\left(p_{0}\right)=300 \mathrm{sec}, \gamma_{1}=0.4, U_{1}=400 \mathrm{~km} / \mathrm{s}$ and $r=3$. Middle panel: Same as left panel for $\kappa_{1}\left(p_{0}\right), 3 \kappa_{1}\left(p_{0}\right)$ and fixed $\delta_{1}, \gamma_{1}, T_{1}\left(p_{0}\right)=200 \mathrm{sec}$, compared with the DSA solution. Other parameters are unchanged from the left panel. Right panel: Spectrum in the case of uniform $\kappa$ and $T$ (with $\left.\kappa_{1}\left(p_{0}\right) / U_{1}^{2} T_{1}\left(p_{0}\right) \gg 1\right)$ for $\kappa_{1}\left(p_{0}\right), 3 \kappa_{1}\left(p_{0}\right)$. Here $T_{1}\left(p_{0}\right)=20$ sec, $U_{1}=100 \mathrm{~km} / \mathrm{s}, \delta_{1}=1, \gamma_{1}=0.4$. The red and green spectra are normalized to 1 at $p=p_{0}$.

Axford, W. I. 1981, in ESA Special Publication, Vol. 161, ESA

Special Publication, ed. S. A. Colgate, 425

Axford, W. I., Leer, E., \& Skadron, G. 1977, International Cosmic

Ray Conference, 11, 132

Band, D., Matteson, J., Ford, L., et al. 1993, ApJ, 413, 281, doi: 10.1086/172995

Bell, A. R. 1978, MNRAS, 182, 147, doi: 10.1093/mnras/182.2.147

-. 2004, MNRAS, 353, 550,

doi: 10.1111/j.1365-2966.2004.08097.x

Blandford, R. D., \& Ostriker, J. P. 1978, ApJL, 221, L29, doi: $10.1086 / 182658$

Brose, R., Telezhinsky, I., \& Pohl, M. 2016, A\&A, 593, A20, doi: 10.1051/0004-6361/201527345

Bruno, A., Bazilevskaya, G. A., Boezio, M., et al. 2018, ApJ, 862, 97, doi: 10.3847/1538-4357/aacc26

Bykov, A. M., Ellison, D. C., Osipov, S. M., \& Vladimirov, A. E. 2014, ApJ, 789, 137, doi: 10.1088/0004-637X/789/2/137

Celli, S., Morlino, G., Gabici, S., \& Aharonian, F. A. 2019, MNRAS, 490, 4317, doi: 10.1093/mnras/stz2897

Cohen, C. M. S., \& Mewaldt, R. A. 2018, Space Weather, 16, 1616, doi: 10.1029/2018SW002006
Cohen, I. J., Schwartz, S. J., Goodrich, K. A., et al. 2019, Journal of Geophysical Research (Space Physics), 124, 3961, doi: 10.1029/2018JA026197

Dalla, S., Marsh, M. S., Kelly, J., \& Laitinen, T. 2013, Journal of Geophysical Research (Space Physics), 118, 5979, doi: 10.1002/jgra.50589

Desai, M. I., Mason, G. M., Dayeh, M. A., et al. 2016, ApJ, 828, 106, doi: 10.3847/0004-637X/828/2/106

Dresing, N., Gómez-Herrero, R., Klassen, A., et al. 2012, Sol. Phys., 281, 281, doi: 10.1007/s11207-012-0049-y

Dröge, W., Kartavykh, Y. Y., Dresing, N., \& Klassen, A. 2016, ApJ, 826, 134, doi: 10.3847/0004-637X/826/2/134

Drury, L. O. 1983, Reports on Progress in Physics, 46, 973, doi: 10.1088/0034-4885/46/8/002

-. 2011, MNRAS, 415, 1807, doi: 10.1111/j.1365-2966.2011.18824.X

Ellison, D. C., \& Ramaty, R. 1985, ApJ, 298, 400, doi: 10.1086/163623

Fairfield, D. H. 1974, J. Geophys. Res. (Space Physics), 79, 1368, doi: 10.1029/JA079i010p01368

Fermi, E. 1949, Physical Review, 75, 1169, doi: 10.1103/PhysRev.75.1169 
Forman, M. A., \& Drury, L. O. 1983, in International Cosmic Ray Conference, Vol. 2, International Cosmic Ray Conference, 267

Fraschetti, F. 2013, ApJ, 770, 84, doi: 10.1088/0004-637X/770/2/84

-. 2014, Nuclear Instruments and Methods in Physics Research A, 742, 169, doi: 10.1016/j.nima.2013.11.066

Fraschetti, F., \& Giacalone, J. 2015, MNRAS, 448, 3555, doi: 10.1093/mnras/stv247

Fraschetti, F., \& Jokipii, J. R. 2011, ApJ, 734, 83, doi: 10.1088/0004-637X/734/2/83

Fraschetti, F., \& Pohl, M. 2017a, MNRAS, 471, 4856, doi: $10.1093 / \mathrm{mnras} / \mathrm{stx} 1833$

Fraschetti, F., \& Pohl, M. 2017b, in European Physical Journal Web of Conferences, Vol. 136, 02009, doi: 10.1051/epjconf/201713602009

Fraschetti, F., Ruffini, R., Vitagliano, L., \& Xue, S. S. 2006, Nuovo Cimento B Serie, 121, 1477, doi: 10.1393/ncb/i2007-10285-x

Giacalone, J. 2005, ApJ, 624, 765, doi: 10.1086/429265

Giacalone, J., \& Jokipii, J. R. 2007, ApJL, 663, L41, doi: $10.1086 / 519994$

Gradshteyn, I. S., Ryzhik, I. M., Jeffrey, A., \& Zwillinger, D. 2007, Table of Integrals, Series, and Products, Academic Press

Jokipii, J. R. 1982, ApJ, 255, 716, doi: 10.1086/159870

—. 1987, ApJ, 313, 842, doi: 10.1086/165022

Jones, F. C., \& Ellison, D. C. 1991, SSRv, 58, 259, doi: 10.1007/BF01206003

Kajdič, P., Blanco-Cano, X., Aguilar-Rodriguez, E., et al. 2012, Journal of Geophysical Research (Space Physics), 117, A06103, doi: 10.1029/2011JA017381

Kang, H., \& Jones, T. W. 2006, Astroparticle Physics, 25, 246, doi: 10.1016/j.astropartphys.2006.02.006

Kennel, C. F., Coroniti, F. V., Scarf, F. L., et al. 1986, J. Geophys. Res. (Space Physics), 91, 11917, doi: 10.1029/JA091iA11p11917

Kis, A., Matsukiyo, S., Otsuka, F., et al. 2018, ApJ, 863, 136, doi: 10.3847/1538-4357/aad08c

Kis, A., Scholer, M., Klecker, B., et al. 2004, Geophys. Res. Lett., 31, L20801, doi: 10.1029/2004GL020759

Krymskii, G. F. 1977, Akademiia Nauk SSSR Doklady, 234, 1306

Lario, D., Berger, L., Decker, R. B., et al. 2019, AJ, 158, 12, doi: 10.3847/1538-3881/ab1e49

Lario, D., Ho, G. C., Decker, R. B., et al. 2003, in American Institute of Physics Conference Series, Vol. 679, Solar Wind Ten, ed. M. Velli, R. Bruno, F. Malara, \& B. Bucci, 640-643, doi: 10.1063/1.1618676

Lee, M. A. 1983, J. Geophys. Res. (Space Physics), 88, 6109, doi: 10.1029/JA088iA08p06109

Lerche, I., \& Schlickeiser, R. 1985, A\&A, 151, 408

Li, G., \& Lee, M. A. 2015, ApJ, 810, 82, doi: 10.1088/0004-637X/810/1/82
Li, G., Zank, G. P., \& Rice, W. K. M. 2003, Journal of Geophysical Research (Space Physics), 108, 1082, doi: 10.1029/2002JA009666

—. 2005, Journal of Geophysical Research (Space Physics), 110, A06104, doi: 10.1029/2004JA010600

Li, J.-T., Ballet, J., Miceli, M., et al. 2018, ApJ, 864, 85, doi: 10.3847/1538-4357/aad598

Malkov, M. A., \& Aharonian, F. A. 2019, ApJ, 881, 2, doi: $10.3847 / 1538-4357 / a b 2 c 01$

Malkov, M. A., Diamond, P. H., \& Sagdeev, R. Z. 2011, Nature Communications, 2, 194, doi: 10.1038/ncomms1195

Malkov, M. A., \& Drury, L. O. 2001, Reports on Progress in Physics, 64, 429, doi: 10.1088/0034-4885/64/4/201

Mason, G. M., Wiedenbeck, M. E., Miller, J. A., et al. 2002, ApJ, 574, 1039, doi: 10.1086/341112

Massaro, E., Perri, M., Giommi, P., \& Nesci, R. 2004, A\&A, 413, 489, doi: 10.1051/0004-6361:20031558

Mewaldt, R. A. 2006, SSRv, 124, 303, doi: 10.1007/s11214-006-9091-0

Mewaldt, R. A., Cohen, C. M. S., Mason, G. M., et al. 2005, in American Institute of Physics Conference Series, Vol. 781, The Physics of Collisionless Shocks: 4th Annual IGPP International Astrophysics Conference, ed. G. Li, G. P. Zank, \& C. T. Russell, 227-232, doi: 10.1063/1.2032701

Mewaldt, R. A., Looper, M. D., Cohen, C. M. S., et al. 2012, SSRv, 171, 97, doi: 10.1007/s11214-012-9884-2

Meyer, M., Horns, D., \& Zechlin, H.-S. 2010, A\&A, 523, A2, doi: 10.1051/0004-6361/201014108

Ng, C. K., \& Reames, D. V. 1994, ApJ, 424, 1032, doi: $10.1086 / 173954$

Pallocchia, G., Laurenza, M., \& Consolini, G. 2017, ApJ, 837, 158, doi: $10.3847 / 1538-4357 / a a 633 a$

Palmer, I. D. 1982, Reviews of Geophysics and Space Physics, 20, 335, doi: 10.1029/RG020i002p00335

Petrosian, V. 2016, ApJ, 830, 28, doi: 10.3847/0004-637X/830/1/28

Pohl, M., Hoshino, M., \& Niemiec, J. 2020, Progress in Particle and Nuclear Physics, 111, 103751, doi: 10.1016/j.ppnp.2019.103751

Ptuskin, V. S., \& Zirakashvili, V. N. 2005, A\&A, 429, 755, doi: 10.1051/0004-6361:20041517

Reville, B., O'Sullivan, S., Duffy, P., \& Kirk, J. G. 2008, MNRAS, 386, 509, doi: 10.1111/j.1365-2966.2008.13059.x

Rice, W. K. M., Zank, G. P., \& Li, G. 2003, Journal of Geophysical Research (Space Physics), 108, 1369, doi: 10.1029/2002JA009756

Schlickeiser, R. 2002, Cosmic Ray Astrophysics, Springer-Verlag Berlin Heidelberg

Schwadron, N. A., Lee, M. A., Gorby, M., et al. 2015, ApJ, 810, 97, doi: 10.1088/0004-637X/810/2/97 
Silberberg, R., \& Tsao, C. H. 1990, Phys. Rep., 191, 351, doi: 10.1016/0370-1573(90)90109-F

Trattner, K. J., Mobius, E., Scholer, M., et al. 1994, J. Geophys. Res. (Space Physics), 99, 13,389, doi: 10.1029/94JA00576

Vainio, R., Pönni, A., Battarbee, M., et al. 2014, Journal of Space Weather and Space Climate, 4, A08, doi: 10.1051/swsc/2014005

Verkhoglyadova, O. P., Li, G., Zank, G. P., Hu, Q., \& Mewaldt, R. A. $2009, A p J, 693,894$, doi: 10.1088/0004-637X/693/1/894

Vladimirov, A., Ellison, D. C., \& Bykov, A. 2006, ApJ, 652, 1246, doi: $10.1086 / 508154$

Voelk, H. J., Morfill, G. E., \& Forman, M. A. 1981, ApJ, 249, 161, doi: $10.1086 / 159272$
Wilson, L. B., I., Koval, A., Szabo, A., et al. 2017, Journal of Geophysical Research (Space Physics), 122, 9115, doi: 10.1002/2017JA024352

Zank, G. P., le Roux, J. A., Webb, G. M., Dosch, A., \& Khabarova, O. 2014, ApJ, 797, 28, doi: 10.1088/0004-637X/797/1/28

Zank, G. P., Rice, W. K. M., \& Wu, C. C. 2000, J. Geophys. Res. (Space Physics), 105, 25079, doi: 10.1029/1999JA000455

Zank, G. P., Hunana, P., Mostafavi, P., et al. 2015, ApJ, 814, 137, doi: 10.1088/0004-637X/814/2/137

Zhao, L., Zhang, M., \& Rassoul, H. K. 2017, ApJ, 836, 31, doi: 10.3847/1538-4357/836/1/31

Zhao, L. L., Zank, G. P., Hu, Q., et al. 2019, ApJL

Zhou, C., Fraschetti, F., Drake, J. J., \& Pohl, M. 2018, Research Notes of the AAS, 2, 145.

http://stacks.iop.org/2515-5172/2/i=3/a=145 